\title{
ASYMPTOTIC REDUCTION OF A CERTAIN BOUNDARY VALUE PROBLEM ARISING FROM THE DENSITY WAVE THEORY OF SPIRAL GALAXIES
}

\author{
By Toshiniko Nishimoto
}

\section{§1. Introduction.}

The dynamical mechanism of the long term maintenance of the spiral structure observed in many disk shaped galaxies has been successfully explored through the density wave theory of the spiral galaxies by C.C. Lin and his collaborators. The mathematical formulation of this theory is based either on the steller dynamic or on the hydrodynamic approach. If we adopt the latter, the basic equation consists of the equation of continuity, the Euler's equations of motion, the equation of state and the Poisson equation. We assume that the basic equation has a steady axisymmetric solution, then we get equations of perturbations (2.1). In the context of the linear theory, there are three levels of approximations of the equations for analysis (private communication of Prof. C. C. Lin): (i) an integro-differential system (2.3a), (2.3b), (2.3c) and (2.4), which is exact as the linearlized equations for the perturbations,

(ii) a third order differential system (2.7a) and (2.7b),

(iii) a second order differential equation (2.9).

Through numerical and asymptotic analyses of the second order differential equation, Lin and his school have arrived at a consistent interpretation of the plysical problems of the spiral galaxies, Lin and Lau [7]. Pannatoni and Lau [10] just begun the study of the integro-differential system by elaborate numerical analysis.

The purpose of this paper is to study the third order differential system of the second level of approximation by the asymptotic analysis and prove that it is asymptotically equivalent to the second order differential equation of the last level, in an appropriate region where the spiral structure prevails.

The program of this paper is as follows. In section 2 , the basic equations and the equation of perturbation of each level of approximations are stated. In section 3, through the block diagonalization technique (Wasow [12], p. 133 ff.), the third order differential system is split into a second order and a first order differential equations. In section 4, we define the so-called admissible regions in which we construct asymptotic expansions of solutions of the third order differential system. And in the last section we consider connection formulas

Recelved December 25, 1979 
between two fundamental systems of solutions defined in two admissible regions, and prove the splitting property, which means that one of the solutions is independent from the other two solutions throughout the region of our interest. In this way, we obtain the main result that the third order differential system with certain boundary conditions is asymptotically equivalent to the second order differential equation with the same boundary conditions. As a consequence, we get an asymptotic formula of the global dispersion relation for the third order differential system.

\section{$\S 2$. Equations of perturbation.}

The basic equation governing the behavior of the steller component are the Boltzmann equation and the Poisson equation for the gravitational potential (Rohlfs [11] Chapter 1). But for simplicity of analysis, a fluid dynamical model has been often adopted for the disk stars. Since the mass distribution in a galaxy is axisymmetrical, we describe the basic equation in the cylindrical coordinate system $(r, \theta, z)$ with the plane $z=0$ coinciding with the plane of symmetry in the disk. we idealize the disk as being infinitesimally thin. Let us denote the surface density, the corresponding gravitational potential, by $\sigma(t, r, \theta), \phi(t, r, \theta, z)$, and the components of velocity of unit mass of the fluid in the $r$-and $\theta$ directions by $u(t, r, \theta), v(t, r, \theta)$ respectively. We can assume that the state of equilibrium of the basic equations $\left\{\sigma_{0}, u_{0}, v_{0}, \phi_{0}\right\}=\left\{\sigma_{0}(r), 0, v_{0}(r), \dot{\phi}_{0}(r)\right\}$ has rotational symmetry, and these quantities characterize models of galaxies. The perturbation to the stationary state $\left\{\tilde{\sigma}_{1}, \tilde{u}_{1}, \tilde{v}_{1}, \tilde{\phi}_{1}\right\}$ satisfies the following set of equations in the linearlized form. [11], [7]:

$$
\begin{aligned}
& \frac{\partial \tilde{\sigma}_{1}}{\partial t}+\Omega \frac{\partial \tilde{\sigma}_{1}}{\partial \theta}+\frac{1}{r}\left\{\frac{\partial}{\partial r}\left(r \sigma_{0} \tilde{u}_{1}\right)+\frac{\partial}{\partial \theta}\left(\sigma_{0} \tilde{v}_{1}\right)\right\}=0 . \\
& \frac{\partial \tilde{u}_{1}}{\partial t}+\Omega \frac{\partial \tilde{u}_{1}}{\partial \theta}-2 \Omega \tilde{v}_{1}=-\left(-\frac{a^{2}}{\sigma_{0}} \frac{\partial \tilde{\sigma}_{1}}{\partial r}+\frac{\partial \tilde{\phi}_{1}}{\partial r}\right), \\
& \frac{\partial \tilde{v}_{1}}{\partial t}+\Omega \frac{\partial \tilde{v}_{1}}{\partial \theta}+\frac{\kappa^{2}}{2 \Omega}-\tilde{u}_{1}=-\frac{1}{r}\left(\frac{a^{2}}{\sigma_{0}} \frac{\partial \tilde{\sigma}_{1}}{\partial \theta}+\frac{\partial \tilde{\phi}_{1}}{\partial \theta}\right), \\
& \frac{\partial^{2} \tilde{\phi}_{1}}{\partial r_{2}}+\frac{1}{r} \frac{\partial \tilde{\phi}_{1}}{\partial r}+\frac{1}{r^{2}} \frac{\partial^{2} \tilde{\phi}_{1}}{\partial \theta^{2}}+\frac{\partial^{2} \tilde{\phi}_{1}}{\partial z^{2}}=4 \pi G \tilde{\sigma}_{1} \delta(z),
\end{aligned}
$$

where $\delta(z)$ is the delta function of Dirac.

Here $\Omega=\Omega(r)$ is the angular velocity $=v_{0}(r) / r, a=a(r)$ is the mean velocity of the gas and $\kappa$ is the epicyclic frequency defined by $2 \Omega(1+r / 2 \Omega d \Omega / d r)^{1 / 2}$. We can assume that these quantities are all given real analytic functions of $r$ and positive. $G$ is the constant of universal gravitation.

We may seek solutions in the form of normal modes having spiral structure 


$$
\begin{aligned}
& \tilde{\sigma}_{1}=\sigma_{1}(r) \exp i(\omega t-m \theta), \\
& \tilde{u}_{1}=u_{1}(r) \exp i(\omega t-m \theta), \\
& \tilde{v}_{1}=v_{1}(r) \exp i(\omega t-m \theta), \\
& \tilde{\phi}_{1}=\phi_{1}(r, z) \exp i(\omega t-m \theta),
\end{aligned}
$$

where $\omega$ is the complex parameter, and $m$ is the number of spiral arms. From (2.1.) and (2.2) we get

$$
\begin{aligned}
& \frac{d u_{1}(r)}{d r}+\left(\frac{1}{r}+\frac{1}{\sigma_{0}} \frac{d \sigma_{0}}{d r}\right) u_{1}(r)-\frac{m \imath v_{1}(r)}{r}+i\left(\frac{\omega-m \Omega}{\sigma_{0}}\right) \sigma_{1}(r)=0, \\
& \frac{\partial\left(\phi_{1}(r, z)+h_{1}(r)\right)}{\partial r}+i(\omega-m \Omega) u_{1}(r)-2 \Omega v_{1}(r)=0,
\end{aligned}
$$

$$
\frac{\kappa^{2}}{2 \Omega} u_{1}(r)+i(\omega-m \Omega) v_{1}(r)-\frac{m \imath}{r}\left(\phi_{1}(r, z)+h_{1}(r)\right)=0,
$$

where

and

$$
h_{1}(r)=\frac{a^{2}(r) \sigma_{1}(r)}{\sigma_{0}(r)},
$$

$$
\left\{\frac{\partial^{2}}{\partial r^{2}}+\frac{1}{r} \frac{\partial}{\partial r}+\frac{\partial^{2}}{\partial z^{2}}-\frac{m^{2}}{r^{2}}\right\} \phi_{1}(r, z)=4 \pi G \sigma_{1}(r) \delta(z) .
$$

The gravitational potential over the disk $\phi_{1}(r)=\phi_{1}(r, 0)$ can be expressed in terms of an integral of the surface mass density

$$
\phi_{1}(r)=-2 \pi G \int_{0}^{\infty} K\left(r, r^{\prime}\right) \sigma_{1}\left(r^{\prime}\right) d r^{\prime},
$$

where $K\left(r, r^{\prime}\right)$ is known (see [7], p. 116).

Equations (2.3a), (2.3b), (2.3c) and (2.4) constitute the integro-differential system which we refer in the introduction as the equations of the first level of approximation.

To apply the asymptotic analysis, the equation (2.3d) connecting the potential $\phi_{1}(r, z)$ with the surface density $\sigma_{1}(r)$ can be approximated for rather tightly wound spirals by the differential relation, Bertin and Mark [1],

$$
\frac{d \phi_{1}(r)}{d r}+\frac{\phi_{1}(r)}{2 r}-\frac{m^{2}-1 / 4}{\imath \Sigma r^{2}} \phi_{1}(r)=\imath \Sigma h_{1}(r),
$$

where

$$
\Sigma(r)=\frac{2 \pi G \sigma_{0}}{a^{2}}=\frac{2 \kappa}{a Q}, \quad Q=\frac{\kappa a}{\pi G \sigma_{0}} .
$$

From the equations (2.3a), (2.3b), (2.3c) and (2.5) we can eliminate $u_{1}(r), v_{1}(r)$ 
and $\sigma_{1}(r)$ and get a second order differential equation for the two variables $\phi_{1}(r)$ and $h_{1}(r)$ in the traditional form [7]

$$
\begin{gathered}
\frac{d^{2} h_{1}}{d r^{2}}+\cdot(A+\imath \Sigma) \frac{d h_{1}}{d r}+\left[B+C-\imath \Sigma\left(\frac{1}{2 r}-A-\frac{\Sigma^{\prime}}{\Sigma}\right)-\frac{m^{2}-1 / 4}{r^{2}}\right] h_{1} \\
=\left[\frac{A}{2 r}-B-\frac{3}{4 r^{2}}\right] \psi_{1},
\end{gathered}
$$

where

$$
\begin{aligned}
& A=-\frac{1}{r} \frac{d \log \mathcal{A}}{d \log r} \quad \mathcal{A}=\frac{\kappa^{2}\left(1-\nu^{2}\right)}{\sigma_{0} r}, \\
& B=-\frac{m^{2}}{r^{2}}+\frac{2 m \Omega}{r^{2} \kappa \nu} \frac{d \log \left(\kappa^{2} / \sigma_{0} \Omega\right)}{d \log r}-\frac{T_{1}}{1-\nu^{2}}+\frac{4 \nu m \Omega}{r^{2} \kappa\left(1-\nu^{2}\right)} \frac{d \log \kappa}{d \log r}, \\
& C=\frac{-\kappa^{2}\left(1-\nu^{2}\right)}{a^{2}}, \\
& \nu=\frac{m(\omega-\Omega)}{\kappa}, \quad T_{1}=-\left(\frac{2 m \Omega}{r \kappa}\right)^{2} \frac{d \log \Omega}{d \log r} .
\end{aligned}
$$

By the standard transformation

$$
\begin{aligned}
& h_{1}=u \exp \left\{-\int^{r} \frac{A+i \Sigma}{2} d r\right\}=u \exp \left\{-\frac{\imath}{2} \int \Sigma d r\right\}\left(\frac{\kappa^{2}\left(1-\nu^{2}\right)}{\sigma_{0} r}\right)^{1 / 2}, \\
& \phi_{1}=v \exp \left\{-\frac{\imath}{2} \int \Sigma d r\right\}\left(\frac{\kappa^{2}\left(1-\nu^{2}\right)}{\sigma_{0} r}\right)^{1 / 2},
\end{aligned}
$$

the equations (2.5) and (2.6) becomes

(2.7a) $\frac{d^{2} u}{d r^{2}}+\left\{B+C+\frac{\Sigma^{2}}{4}-\left(\frac{A^{2}}{4}+\frac{A^{\prime}}{2}\right)-\frac{\imath \Sigma}{2}\left(\frac{1}{r}-A-\frac{\Sigma^{\prime}}{\Sigma}\right)-\frac{m^{2}-1 / 4}{r^{2}}\right) u$

$$
+\left\{-\frac{A}{2 r}+B+\frac{3}{4 r^{2}}\right\} v=0
$$

(2.7b) $\frac{d v}{d r}-\left\{\frac{A+\imath \Sigma}{2}-\frac{1}{2 r}+\frac{m^{2}-1 / 4}{\imath \Sigma r^{2}}\right\} v-\imath \Sigma u=0$.

The above equations (2.7a) and (2.7b) constitute a third order differential system of the second level of approximation which we shall study in this paper.

From the basis of the physical consideration, Lau and Mark [4] reduced the equations (2.7a) and (2.7b) by replacing $v$ in $(2.7 \mathrm{a})$ by $-2 u$ and arrived at a second order differential equation

$$
\frac{d^{2} u}{d r^{2}}+\left\{-B+C+\frac{\Sigma^{2}}{4}-\left(\frac{A^{2}}{4}-\frac{A}{r}+\frac{A^{\prime}}{2}\right)\right.
$$




$$
\left.-\imath \frac{\Sigma}{2}\left(\frac{1}{r}-A-\frac{\Sigma^{\prime}}{\Sigma}\right)-\left(\frac{m^{2}}{r^{2}}+\frac{5}{4 r^{2}}\right)\right\} u=0
$$

which can also be rewritten as (see section 3 for details)

$$
\begin{aligned}
\frac{d^{2} u}{d r^{2}}+K^{2} u=0, \\
K^{2}=k^{2}+R, \\
k^{2}=\left(\frac{\kappa}{a}\right)^{2}\left(\frac{1}{Q^{2}}-1+\nu^{2}\right), \quad Q=\frac{\kappa a}{\pi G \sigma_{0}}, \\
R=-\imath \frac{\Sigma}{2} \frac{d}{d r} \log \left[Q^{2}\left(1-\nu^{2}\right)\right]+\frac{T_{1}}{1-\nu^{2}}-\frac{2 m \Omega}{r^{2} \kappa \nu} \frac{d \log \left(\kappa^{2} / \sigma_{0} \Omega\right)}{d \log r} \\
\quad-\frac{4 \nu m \Omega}{\kappa r^{2}\left(1-\nu^{2}\right)} \frac{d \log \kappa}{d \log r}-\left\{\frac{\kappa^{2}\left(1-\nu^{2}\right)^{1 / 2}}{\sigma_{0} r}\right\} \frac{d^{2}}{d r^{2}}\left(\frac{\sigma_{0} r}{\kappa^{2}\left(1-\nu^{2}\right)}\right)^{1 / 2} \\
\quad+\frac{\kappa^{2}\left(1-\nu^{2}\right)}{\sigma_{0} r^{2}}-\frac{d}{d r}\left(\frac{\sigma_{0} r}{\kappa^{2}\left(1-\nu^{2}\right)}\right)-\frac{5}{4 r^{2}} \cdot
\end{aligned}
$$

Equation (2.9) is of the third level of approximation that has been extensively studied, for example Lin and Lau [5], [7] and Nishimoto [9].

\section{§ 3. Asymptotic reduction.}

In this section, we construct a linear transformation which asymptotically splits the differential system (2.7a) and (2.7b) into a second order differential equation of single variable and a first order differential equation of another variable. To do so, we rewrite the equations $(2.7 \mathrm{a})$ and $(2.7 \mathrm{~b})$ by using the physical quantities and introduce a large parameter $\lambda$ in order to apply the asymptotic method.

In the equation (2.7a) and (2.7b), we replace $A, B$ and $C$ by their difinitions written below the equation (2.6).

Since

$$
\begin{aligned}
& A=-\frac{1}{r} \frac{d \log \mathcal{A}}{d \log r}=\frac{1}{r} \frac{d \log \mathcal{A}^{-1}}{d \log r}=\frac{1}{r} \frac{r}{\phi^{-1}} \frac{d \mathcal{A}^{-1}}{d r}=\frac{\kappa^{2}\left(1-\nu^{2}\right)}{\sigma_{0} r} \frac{d}{d r}\left(\frac{\sigma_{0} r}{\kappa^{2}\left(1-\nu^{2}\right)}\right), \\
& A^{\prime}=-\left(\frac{\sigma_{0} r}{\kappa^{2}\left(1-\nu^{2}\right)}\right)^{-2}\left\{\frac{d}{d r}\left(\frac{\sigma_{0} r}{\kappa^{2}\left(1-\nu^{2}\right)}\right)\right\}^{2}+\left(\frac{\sigma_{0} r}{\kappa^{2}\left(1-\nu^{2}\right)}\right)^{-1} \frac{d^{2}}{d r^{2}}\left(\frac{\sigma_{0} r}{\kappa^{2}\left(1-\nu^{2}\right)}\right),
\end{aligned}
$$

it is easily verified that

$$
\frac{A^{2}}{4}+\frac{A^{\prime}}{2}=\left(\frac{\kappa^{2}\left(1-\nu^{2}\right)}{\sigma_{0} r}\right)^{1 / 2} \frac{d^{2}}{d r^{2}}\left(\frac{\sigma_{0} r}{\kappa^{2}\left(1-\nu^{2}\right)}\right)^{1 / 2},
$$


and since

$$
\begin{aligned}
& \int_{r_{0}}^{r}\left(\frac{1}{r}-A-\frac{\Sigma^{\prime}}{\Sigma}\right) d r=[\log r+\log \mathcal{A}-\log \Sigma]_{r_{0}}^{r} \\
= & {\left[\log r\left(\frac{\sigma_{0} r}{\kappa^{2}\left(1-\nu^{2}\right)}\right)^{-1} \frac{a_{0} Q}{2 \kappa}\right]_{r_{0}}^{r}=\left[\log Q\left(1-\nu^{2}\right) \frac{\kappa a}{2 \sigma_{0}}\right]_{r_{0}}^{r}, }
\end{aligned}
$$

then we have

$$
\frac{1}{r}-A-\frac{\Sigma^{\prime}}{\Sigma}=\frac{d}{d r} \log \left[Q^{2}\left(1-\nu^{2}\right)\right]
$$

And we have

$$
C+\frac{\Sigma^{2}}{4}=\left(\frac{\kappa}{a}\right)^{2}\left(\frac{1}{Q^{2}}-1+\nu^{2}\right) .
$$

Thus we have

$$
\frac{d^{2} u}{d r}+\left(k_{1}^{2}+R_{1}\right) u+R_{2} v=0,
$$

$$
\frac{d v}{d r}+k_{2} u+\left(k_{3}+R_{3}\right) v=0,
$$

where

$$
\begin{aligned}
k_{1}^{2}= & \frac{\kappa^{2}}{a^{2}}\left(\frac{1}{Q^{2}}-1+\nu^{2}\right), \\
k_{2}= & -\imath \Sigma=-\imath \frac{2 \pi G \sigma_{0}}{a^{2}}=-\frac{2 i \kappa}{a Q}, \quad k_{3}=-\frac{i \Sigma}{2}=-\frac{\imath \kappa}{a Q} \\
R_{1}= & -\frac{\imath \Sigma}{2} \frac{d}{d r}\left[\log \left\{Q^{2}\left(1-\nu^{2}\right)\right\}\right]-\frac{T_{1}}{1-\nu^{2}}+\frac{2 m \Omega}{r^{2} \kappa \nu} \frac{d \log \left(\kappa^{2} / \sigma_{0} \Omega\right)}{d \log r} \\
& +\frac{4 \nu m \Omega}{r^{2} \kappa\left(1-\nu^{2}\right)} \frac{d \log \kappa}{d \log r}-\left(\frac{\kappa^{2}\left(1-\nu^{2}\right)}{\sigma_{0} r}\right)^{1 / 2} \frac{d^{2}}{d r^{2}}\left(\frac{\sigma_{0} r}{\kappa^{2}\left(1-\nu^{2}\right)}\right)^{1 / 2} \\
& -\frac{2 m^{2}-1 / 4}{r^{2}}, \quad \\
R_{2}= & -\frac{\kappa^{2}\left(1-\nu^{2}\right)}{2 \sigma_{0} r} \frac{d}{d r}\left(\frac{\sigma_{0} r}{\kappa^{2}\left(1-\nu^{2}\right)}\right)-\frac{T_{1}}{1-\nu^{2}}+\frac{2 m \Omega}{r^{2} \kappa \nu}-\frac{d \log \left(\kappa^{2} / \sigma_{0} \Omega\right)}{d \log r} \\
& +\frac{4 \nu m \Omega}{r^{2} \kappa\left(1-\nu^{2}\right)} \frac{d \log \kappa}{d \log r}+\frac{3-4 m^{2}}{4 r^{2}}, \\
R_{3}= & \frac{1}{2 r}-\frac{\kappa^{2}\left(1-\nu^{2}\right)}{\sigma_{0} r} \frac{d}{d r}\left(\frac{\sigma_{0} r}{\kappa^{2}\left(1-\nu^{2}\right)}\right)+\frac{a Q\left(m^{2}-1 / 4\right)}{2 \imath r^{2} \kappa} .
\end{aligned}
$$


Now we introduce a dimensionless large parameter $\lambda$ which is a typical value of $\kappa r / a$. For a realistic model of galaxies, $\lambda$ is about 32. The orders of magnitude of the coefficients $k_{\imath}(\imath=1,2,3)$ and $R_{\imath}(\imath=1,2,3)$ on the region where spiral structure prevails are assumed to be (this is the case for tightly wound normal spiral galaxies, [4] and [7])

$$
\begin{array}{ll}
k_{\imath}=0(\lambda), \quad(i=1,2,3), & \\
R_{1}=0(\lambda), \quad R_{2}=0(\lambda), \quad R_{3}=0(1) .
\end{array}
$$

By using the large parameter $\lambda$, the equation (3.1) is rewritten in a simpler notation as

$$
\begin{aligned}
& u^{\prime \prime}-\lambda^{2}\left\{\left(a_{0}^{2}+\frac{a_{1}}{\lambda}\right) u+\frac{b_{1}}{\lambda} v\right\}=0, \\
& v^{\prime}-\lambda\left\{c_{0} u+\left(d_{0}+\frac{d_{1}}{\lambda}\right) v\right\}=0,
\end{aligned}
$$

or if we put

$$
u_{1}=u, \quad u_{2}=\varepsilon u^{\prime}, \quad u_{3}=v \quad \text { and } \varepsilon=\lambda^{-1},
$$

then the above equation becomes

$$
\varepsilon\left(\begin{array}{l}
u_{1} \\
u_{2} \\
u_{3}
\end{array}\right)^{\prime}=\left\{\left(\begin{array}{ccc}
0 & 1 & 0 \\
a_{0}^{2} & 0 & 0 \\
c_{0} & 0 & d_{0}
\end{array}\right)+\varepsilon\left(\begin{array}{ccc}
0 & 0 & 0 \\
a_{1} & 0 & b_{1} \\
0 & 0 & d_{1}
\end{array}\right)\right)\left(\begin{array}{l}
u_{1} \\
u_{2} \\
u_{3}
\end{array}\right),
$$

where

$$
\begin{array}{lll}
a_{0}^{2}=-\lambda^{-2} k_{1}^{2}, & a_{1}=-\lambda^{-1} R_{1}, & b_{1}=-\lambda^{-1} R_{2}, \\
c_{0}=-\lambda^{-1} k_{2}, & d_{0}=-\lambda^{-1} k_{3}, & d_{1}=-\lambda^{-1} R_{3} .
\end{array}
$$

Let us examine singularities and turning points of the above equation (3.2) for real $\omega$ and $r$. The singular points are

and the points where

$$
r=0, \text { the center of galaxy, }
$$

$$
\nu \equiv \frac{m(\omega-\Omega)}{\kappa}=0,+1,-1
$$

The latter are usually designated as the corotation resonance $r_{c o}$, the outer Lindbladt resonance $r_{O L R}$ and the inner Lindbladt resonance $r_{I L R}$. The function $a_{1}(r)$ has poles of order two at $r=0$ and $r_{O L R}, r_{I L R}$ and a simple pole at $r_{c o}$. The function $b_{1}(r)$ also has a pole of order two at $r=0$ and simple poles at $r_{O L R}, r_{I L R}$ and $r_{c o}$, and the function $d_{1}(x)$ has a pole of order two at $r=0$, simple poles at $r_{O L R}, r_{I L R}$. 
The turning points are those at which roots of the equation

$$
f(\mu) \equiv \operatorname{det}\left\{\left(\begin{array}{ccc}
0 & 1 & 0 \\
a_{0}^{2} & 0 & 0 \\
c_{0} & 0 & d_{0}
\end{array}\right)-\mu E\right\}=0
$$

coalesce. Since we have $f(\mu)=\left(\mu-d_{0}\right)\left(\mu^{2}-a_{0}^{2}\right)$, turning points are those at which either

$$
a_{0}^{2} \equiv-\lambda^{-2}\left(\frac{\kappa}{a}\right)^{2}\left(Q^{-2}-1+\nu^{2}\right)=0
$$

or

$$
a_{0}^{2}-d_{0}^{2} \equiv \lambda^{-2}\left(\frac{\kappa}{a}\right)^{2}\left(1-\nu^{2}\right)=0
$$

Thus the Lindbladt resonance $r_{O L R}, r_{I L R}$ where $\nu^{2}=1$ are simple turning points. From the numerical investigations, we reasonably assume that the function $a_{0}^{2}(r)$ has a double zero at $r_{c o}$ where $\nu=0$ and a simple zero at $r_{c e}$ such that $r_{I L R}<r_{c e}<r_{c 0}$. Thus for real $\omega$, the function $a_{0}^{2}(r)$ is real and has a curve like Fig. 1, [7].

As is easily verified, the point $r=0$ is the irregular singular point and other poles are regular singular points of the differential system (3.2). Thus we are concerned with the equation having an irregular singular point, regular singular points and turning points. But at present, there is no global theory which is

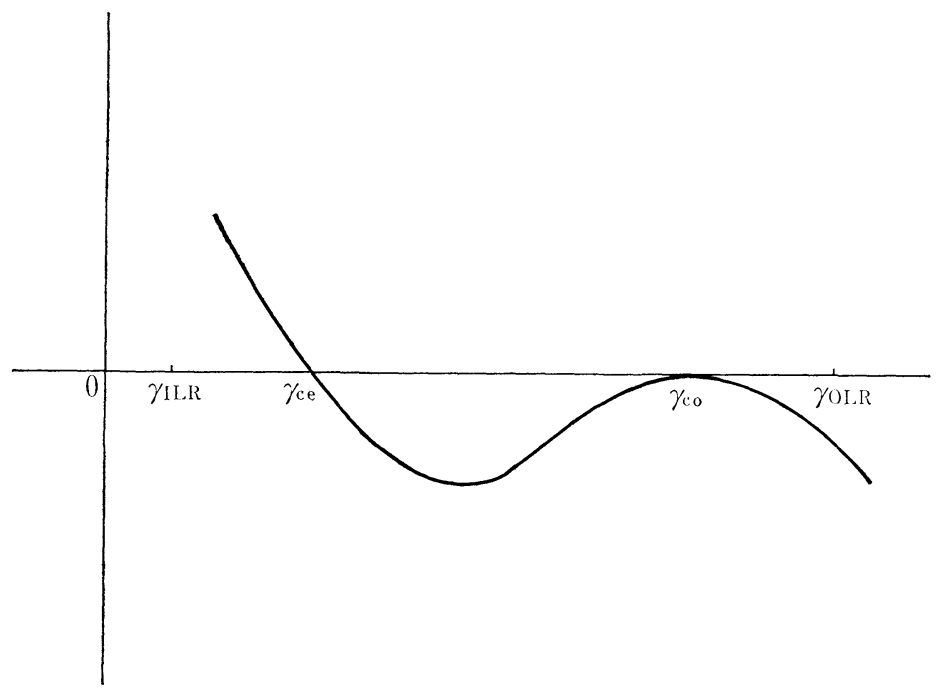

Fig. 1. Function $a_{0}^{2}(r)$ for real $\omega$ 
available to our equation, and so we restrict our attention to some subregion, for example, to a neighborhood of $r_{c o}, r_{I L R}, r_{O L R}$ or a segment between $r_{c e}$ and $r_{c o}$. just like other papers [4], [6], [7] and Mark [8].

Now we apply the following transformation to the equation (3.2).

$$
U=\left(P_{0}+\varepsilon P_{1}\right) W
$$

where

$$
\begin{aligned}
& U=\left(\begin{array}{l}
u_{1} \\
u_{2} \\
u_{3}
\end{array}\right), \quad W=\left(\begin{array}{l}
w_{1} \\
w_{2} \\
w_{3}
\end{array}\right), \quad P_{0}=\left(\begin{array}{ccc}
1 & 0 & 0 \\
0 & 1 & 0 \\
\frac{c_{0} d_{0}}{a_{0}^{2}-d_{0}^{2}} & \frac{c_{0}}{a_{0}^{2}-d_{0}^{2}} & 1
\end{array}\right) \\
& P_{1}=\left(\begin{array}{lll}
\frac{-b_{1} c_{0} d_{0}}{\left(a_{0}^{2}-d_{0}^{2}\right)^{2}} & \frac{-b_{1} c_{0}}{\left(a_{0}^{2}-d_{0}^{2}\right)^{2}} & \frac{-b_{1}}{a_{0}^{2}-d_{0}^{2}} \\
\frac{-b_{1} c_{0} d_{0}^{2}}{\left(a_{0}^{2}-d_{0}^{2}\right)^{2}} & \frac{-b_{1} c_{0} d_{0}}{\left(a_{0}^{2}-d_{0}^{2}\right)^{2}} & \frac{-b_{1} d_{0}}{a_{0}^{2}-d_{0}^{2}} \\
\frac{a_{0}^{2} \alpha_{2}+d_{0} \alpha_{1}}{a_{0}^{2}-d_{0}^{2}} & \frac{\alpha_{1}+d_{0} \alpha_{2}}{a_{0}^{2}-d_{0}^{2}} & 0
\end{array}\right), \\
& \alpha_{1}=\frac{c_{0}\left(d_{0} d_{1}-a_{1}\right)}{a_{0}^{2}-d_{0}^{2}}-\frac{3 b_{1} c_{0}^{2} d_{0}}{\left(a_{0}^{2}-d_{0}^{2}\right)^{2}}+\left(\frac{c_{0} d_{0}}{a_{0}^{2}-d_{0}^{2}}\right)^{\prime}, \\
& \alpha_{2}=\frac{c_{0} d_{0}}{a_{0}^{2}-d_{0}^{2}}-\frac{b_{1} c_{0}^{2}}{\left(a_{0}^{2}-d_{0}^{2}\right)^{2}}+\left(\frac{c_{0}}{a_{0}^{2}-d_{0}^{2}}\right)^{\prime} .
\end{aligned}
$$

Then the resultant equation is after some calculations

$$
\varepsilon \frac{d W}{d r}=\left\{G_{0}(r)+\varepsilon G_{1}(r)+\varepsilon^{2} G_{2}(r, \varepsilon)\right\} W,
$$

where

$$
G_{0}(r)=\left(\begin{array}{ccc}
0 & 1 & 0 \\
a_{0}^{2} & 0 & 0 \\
0 & 0 & d_{0}
\end{array}\right), \quad G_{1}(r)=\left(\begin{array}{ccc}
\frac{b_{1} c_{0}}{a_{0}^{2}-d_{0}^{2}} & 0 & 0 \\
a_{1}+\frac{b_{1} c_{0} d_{0}}{a_{0}^{2}-d_{0}^{2}} & 0 & 0 \\
0 & 0 & d_{1}-\frac{b_{1} b_{0}}{a_{0}^{2}-d_{0}^{2}}
\end{array}\right),
$$

and $G_{2}(r, \varepsilon)$ is a polynomial of $\varepsilon$ of degree 3 and its coefficients are rational functions of $a_{0} a_{1} b_{1} c_{0} d_{0}$ and $d_{1}$. If we write down the expression of $G_{2}(r, \varepsilon)$, it is easy to count the order of poles at each singularity. We don't go into the details here about this point, but only remark that the remainder term $G_{2}(r, \varepsilon) \varepsilon^{2}$ can be written in the neighborhood of each singularity as 


$$
G_{2}(r, \varepsilon) \varepsilon^{2}=\sum_{\imath=2}^{5} G_{\imath}^{(l)}(r)\left\{\tau^{(l)}(r) \varepsilon\right\}^{\imath},
$$

where $l$ denotes one of singularities $0, r_{I L R}, r_{c o}$ or $r_{O L R}, G_{l}^{(l)}(r)$ is bounded in the neighborhood of a singular point $l$ and $\tau^{(l)}(r)$ is such that

$$
\tau^{(l)}(r)= \begin{cases}r^{-2} & \text { for } l=0, \\ \left(r-r_{I L R}\right)^{-3} & \text { for } l=r_{I L R}, \\ \left(r-r_{c o}\right)^{-1} & \text { for } l=r_{c o}, \\ \left(r-r_{O L R}\right)^{-3} & \text { for } l=r_{O L R} .\end{cases}
$$

Details of the construction of (3.3) and (3.4) are given in Appendix.

Before constructing solutions of equation (3.4), let us compare it with the second order equation (2.9). If we neglect the terms of order higher than $O(\varepsilon)$ in the equation (3.4), then we have

$$
\begin{aligned}
& \varepsilon \frac{d}{d r}\left[\begin{array}{l}
\hat{w}_{1} \\
\hat{w}_{2}
\end{array}\right]=\left\{\left[\begin{array}{cc}
0 & 1 \\
a_{0}^{2} & 0
\end{array}\right]+\varepsilon\left[\begin{array}{ll}
g_{1} & 0 \\
g_{2} & 0
\end{array}\right]\right\}\left[\begin{array}{l}
\hat{w}_{1} \\
\hat{w}_{2}
\end{array}\right], \\
& \varepsilon \frac{d \hat{w}_{3}}{d r}=\left\{d_{0}+\varepsilon g_{3}\right\} \hat{w}_{3},
\end{aligned}
$$

where

$$
g_{1}=\frac{b_{1} c_{0}}{a_{0}^{2}-d_{0}^{2}}, \quad g_{2}=a_{1}+\frac{b_{1} c_{0} d_{0}}{a_{0}^{2}-d_{0}^{2}}, \quad g_{3}=d_{1}-\frac{b_{1} c_{0}}{a_{0}^{2}-d_{0}^{2}}
$$

The equation (3.5a) is equivalent to

$$
\begin{aligned}
& \varepsilon^{2} \frac{d^{2} w}{d r^{2}}-\left\{a_{0}^{2}+\varepsilon g_{2}+\varepsilon^{2}\left(\frac{g_{1}^{\prime}}{2}+\frac{g_{1}^{2}}{4}-\right)\right\} w=0, \\
& w=\hat{w}_{1} \exp \left\{-\int \frac{g_{1}}{2} d r\right\} .
\end{aligned}
$$

Since we have (see the notations below (3.2))

$$
\begin{aligned}
& \varepsilon^{-2} a_{0}^{2}=-\left(\frac{\kappa}{a}\right)^{2}\left(\frac{1}{Q^{2}}-1+\nu^{2}\right)=-k_{1}^{2}=-k^{2}, \\
& \varepsilon^{-1} g_{2}=\lambda g_{2}=\lambda\left\{a_{1}-\frac{2 b_{1}}{\left(1-\nu^{2}\right) Q^{2}}\right\}=-\left\{R_{1}-\frac{2 R_{2}}{\left(1-\nu^{2}\right) Q^{2}}\right\},
\end{aligned}
$$

then both equations (2.9) and (3.6) have the same principal term, and since the quantity $\left(1-\nu^{2}\right) Q^{2}$ is nearly one in the neighborhoods of $r_{c e}$ and $r_{c o}$, the remainder term $R$ in the equation (2.9) may be a good approximation of $-\varepsilon^{-1} g_{2}$ on a neighborhood of the segment between $r_{c e}$ and $r_{c o}$ where it is the most important 
region for the wave mechanism of the density waves.

Now, we consider boundary conditions for the equation (3.4). The boundary conditions for the equation (2.9) are such that

(i) $u(r)$ exponentially decays for $r<r_{c e}$,

(ii) $e^{\imath \omega t} u(r)$ represents an outgoing wave for $r>r_{c o}$.

The physical reasons for these choices of the boundary conditions are given in [7].

Since the equation (3.4) may be approximated by (3.5a), (3.5b) and the equation (3.5a) is essentially equivalent to the equation (2.9), then it seems quite reasonable to put the boundary conditions (3.7) for the equation (3.4).

In the next section, we shall construct asymptotic expansion of solutions of the equations (3.4) and (3.5), and show that the solutions of (3.5) really approximate the solutions of (3.4) in appropriate regions of the complex $r$-plane. From these facts, we can say that the boundary conditions (3.7) bring us a global dispersion relation or a quantum condition for our system (3.4) and (3.1) which is analogous to the one for the second order equation, [6], [9].

\section{$\S 4$. Asymptotic solutions.}

By the transformation,

$$
W=T_{0} X, \quad T_{0}=\left(\begin{array}{ccc}
1 & -1 & 0 \\
a_{0} & a_{0} & 0 \\
0 & 0 & 1
\end{array}\right), \quad X=\left(\begin{array}{l}
x_{1} \\
x_{2} \\
x_{3}
\end{array}\right)
$$

the equation (3.4) becomes

$$
\varepsilon \frac{d X}{d r}=\left\{\tilde{H}_{0}(r)+\varepsilon \widetilde{H}_{1}(r)+\varepsilon^{2} \widetilde{H}_{2}(r, \varepsilon)\right\} X
$$

with

where

$$
\begin{aligned}
& \tilde{H}_{0}(r)=\operatorname{diag}\left\{a_{0}(r),-a_{0}(r), d_{0}(r)\right\}, \\
& \tilde{H}_{1}(r)=\left(\begin{array}{ccc}
h_{11}^{(1)} & h_{12}^{(1)} & 0 \\
h_{21}^{(1)} & h_{22}^{(1)} & 0 \\
0 & 0 & g_{3}
\end{array}\right),
\end{aligned}
$$

$$
\begin{aligned}
& h_{11}^{(1)}=\frac{1}{2}\left\{g_{1}+\frac{g_{2}}{a_{0}}-\frac{a_{0}^{\prime}}{a_{0}}\right\}=-\frac{a_{0}^{\prime}}{2 a_{0}}+\frac{a_{1}}{2 a_{0}}+\frac{b_{1} c_{0}}{2 a_{0}\left(a_{0}-d_{0}\right)}, \\
& h_{12}^{(1)}=-\frac{1}{2}\left\{g_{1}+\frac{g_{2}}{a_{0}}+\frac{a_{0}^{\prime}}{a_{0}}\right\}=-\frac{a_{0}^{\prime}}{2 a_{0}}-\frac{a_{1}}{2 a_{0}}-\frac{b_{1} c_{0}}{2 a_{0}\left(a_{0}-d_{0}\right)},
\end{aligned}
$$




$$
\begin{aligned}
& h_{21}^{(1)}=\frac{1}{2}\left\{-g_{1}+\frac{g_{2}}{a_{0}}-\frac{a_{0}^{\prime}}{a_{0}}\right\}=-\frac{a_{0}^{\prime}}{2 a_{0}}+\frac{a_{1}}{2 a_{0}}-\frac{b_{1} c_{0}}{2 a_{0}\left(a_{0}+d_{0}\right)}, \\
& h_{22}^{(1)}=\frac{1}{2}\left\{g_{1}-\frac{g_{2}}{a_{0}}-\frac{a_{0}^{\prime}}{a_{0}}\right\}=-\frac{a_{0}^{\prime}}{2 a_{0}}-\frac{a_{1}}{2 a_{0}}+\frac{b_{1} c_{0}}{2 a_{0}\left(a_{0}+d_{0}\right)} .
\end{aligned}
$$

The remainder term $\tilde{H}_{2}(r, \varepsilon) \varepsilon^{2}$ has singularities at $r=0, r_{I L R}, r_{c e}, r_{c o}$ and $r_{O L R}$, and can be written

$$
\varepsilon^{2} \widetilde{H}_{2}(r, \varepsilon)=\sum_{\imath=2}^{5} \widetilde{H}_{\imath}^{(l)}(r)\left\{\sigma^{(l)}(r) \varepsilon\right\}^{\imath} .
$$

where $\widetilde{H}_{\imath}^{(l)}(r)$ is bounded at singularities, and $\sigma^{(l)}(r)$ is defined as

$$
\sigma^{(l)}(r)= \begin{cases}\tau^{(l)}(r) & \text { for } l=0, r_{I L R}, \quad r_{O L R}, \\ \left(r-r_{c e}\right)^{-1 / 2} & \text { for } l=r_{c e}, \\ \left(r-r_{c o}\right)^{-2} & \text { for } l=r_{c o} .\end{cases}
$$

If we apply the same transformation (4.1) to the equation (3.5a), (3.5b), we get te equation (4.2) with $\widetilde{H}_{2}(r, \varepsilon) \varepsilon^{2} \equiv 0$.

We further change the equation (4.2) by the transformation

$$
X=\left\{E+\varepsilon T_{1}\right\} Y, \quad Y=\left(\begin{array}{c}
y_{1} \\
y_{2} \\
y_{3}
\end{array}\right)
$$

where $E$ is the 3 -dim unit matrix, and $T_{1}$ is

$$
T_{1}=\frac{1}{2 a_{0}}\left(\begin{array}{ccc}
g_{1}+\frac{a_{0}^{\prime}}{a_{0}}, & \frac{g_{2}}{a_{0}}+\left(g_{1}+\frac{a_{0}^{\prime}}{a_{0}}\right), & 0 \\
\frac{g_{2}}{a_{0}}-\left(g_{1}+\frac{a_{0}^{\prime}}{a_{0}}\right), & -\left(g_{1}+\frac{a_{0}^{\prime}}{a_{0}}\right), & 0 \\
0, & 0, & 0
\end{array}\right) .
$$

By assuming that

$$
\left|\varepsilon g_{2} / 2 a_{0}^{2}\right| \ll 1
$$

we expand $\left\{\operatorname{det}\left(E+\varepsilon T_{1}\right)\right\}^{-1}=\left\{1-\varepsilon^{2} g_{2}^{2} / 4 a_{0}^{4}\right\}^{-1}$ in power series of $\varepsilon$, and obtain from (4.2) and (4.3)

$$
\varepsilon \frac{d Y}{d r}=\left\{H_{0}(r)+\varepsilon H_{1}(r)+\varepsilon^{2} H_{2}(r, \varepsilon)\right\} Y
$$

Here $H_{0}(r)=\tilde{H}_{0}(r), H_{1}(r)=\operatorname{diag} \tilde{H}_{1}(r)$ and $\varepsilon^{2} H_{2}(r, \varepsilon)$ is a power series of $\varepsilon$ such that

$$
\varepsilon^{2} H_{2}(r, \varepsilon)=\sum_{\imath=2}^{\infty} H_{\imath}^{(l)}(r)\left\{\mu^{(l)}(r) \varepsilon\right\}^{\imath} .
$$


where $H_{\imath}^{(l)}(r)$ are bounded at singularities $r=l$ and $\mu^{(l)}(r)$ is such that

$$
\mu^{(l)}(r)= \begin{cases}\tau^{(l)}(r) & \text { for } l=0, r_{I L R}, r_{O L R}, \\ \left(r-r_{c e}\right)^{-3 / 2} & \text { for } l=r_{c e}, \\ \left(r-r_{c o}\right)^{-3} & \text { for } l=r_{c o},\end{cases}
$$

Now we define a diagonal matrix $\Lambda(r, \varepsilon)$ by

$$
\Lambda(r, \varepsilon) \equiv \operatorname{diag}\left\{\Lambda_{1}(r, \varepsilon), \Lambda_{2}(r, \varepsilon), \Lambda_{3}(r, \varepsilon)\right\} \equiv \int^{r}\left\{\frac{H_{0}(r)}{\varepsilon}+H_{1}(r)\right\} d r,
$$

and put

$$
Y(r, \varepsilon)=\{E+Z(r, \varepsilon)\} \exp \Lambda(r, \varepsilon),
$$

then $Z(r, \varepsilon)$ satisfies

$$
\varepsilon \frac{d Z}{d r}=\left(H_{0}+\varepsilon H_{1}\right) Z-Z\left(H_{0}+\varepsilon H_{1}\right)+\varepsilon^{2} H_{2}(E+Z),
$$

where $Z$ is a 3 by 3 matrix with elements $z_{\imath \jmath}(i, j=1,2,3)$. If we denote entries of the matrix $H_{2}(r, \varepsilon)$ by $h_{\imath \jmath}(r, \varepsilon)(i, \jmath=1,2,3)$, then $z_{\imath \jmath}$ satisfy-equations

$$
\begin{array}{r}
\varepsilon \frac{d z_{\imath \jmath}}{d r}=\left\{h_{i}(r, \varepsilon)-h_{\jmath}(r, \varepsilon)\right\} z_{\imath \jmath}+\varepsilon^{2}\left\{h_{\imath \jmath}(r, \varepsilon)+\sum_{k=1}^{3} h_{i k}(r, \varepsilon) z_{k \jmath}\right\}, \\
(i, j=1,2,3) .
\end{array}
$$

Here we denote the diagonal elements of the matrix $H_{0}+\varepsilon H_{1}$ by $h_{i}(r, \varepsilon)$ :

$$
\begin{aligned}
& h_{1}(r, \varepsilon)=a_{0}+\varepsilon h_{11}^{(1)}=a_{0}+\varepsilon\left\{-\frac{a_{0}^{\prime}}{2 a_{0}}+\frac{a_{1}}{2 a_{0}}+\frac{b_{1} c_{0}}{2 a_{0}\left(a_{0}-d_{0}\right)}\right\}, \\
& h_{2}(r, \varepsilon)=-a_{0}+\varepsilon h_{22}^{(1)}=-a_{0}+\varepsilon\left\{-\frac{a_{0}^{\prime}}{2 a_{0}}-\frac{a_{1}}{2 a_{0}}+\frac{b_{1} c_{0}}{2 a_{0}\left(a_{0}+d_{0}\right)}\right\}, \\
& h_{3}(r, \varepsilon)=d_{0}+\varepsilon g_{3}=d_{0}+\varepsilon\left\{d_{1}-\frac{b_{1} c_{0}}{a_{0}^{2}-d_{0}^{2}}\right\} .
\end{aligned}
$$

The differential systems (4.9) are equivalent to systems of integral equations

$$
\begin{aligned}
z_{\imath j}(r, \varepsilon)= & \varepsilon \int_{C_{\imath \jmath}}^{r}\left[\exp \left\{\gamma_{\imath \jmath}(r, \varepsilon)-\gamma_{\imath j}(s, \varepsilon)\right\}\right] \\
& \cdot\left\{h_{\imath \jmath}(s, \varepsilon)+\sum_{k=1}^{3} h_{i k}(s, \varepsilon) z_{k j}(s, \varepsilon)\right\} d s, \\
\gamma_{\imath \jmath}(r, \varepsilon)= & \frac{1}{\varepsilon}\left\{\int_{C_{\imath}}^{r} h_{i}(r, \varepsilon) d r-\int_{C_{\jmath}}^{r} h_{j}(r, \varepsilon) d r\right\},
\end{aligned}
$$


where paths of integral $C_{\imath \jmath}$ and $C_{\imath}$ are specified in later.

Now let us consider $r$ as the complex variable, and solve the integral equation (4.11) in a certain bounded region $\Pi$ in the complex $r$-plane. Then we assume that each coefficient in the equation (3.2) is extended to be analytic function on $\Pi$ except singular points stated in section 3 . The domain $\Pi$ is a neighborhood of the segment between $r_{c e}$ and $r_{c o}$ and does not contain the Lindbladt resonances $r_{I L R}, r_{O L R}$ and $r=0$. Hitherto, we assumed that the turning points $r_{c e}$ and $r_{c o}$ are on the real axis. For $\omega$ complex but nearly real, $r_{c e}$ or $r_{c o}$ may be complex but nearly real and the general nature of the problem is not greatly changed. Thus we assume that $\Pi$ contains $r_{c e}$ and $r_{c o}$ in its interior and contains no other turning points or singular points. The reasons why we choose such a region $\Pi$ are the followings:

(1) From the earlier works, the wave mechanism of density waves is the most important in this region, [7] and [9].

(2) The original differential system (3.1) or (3.2) has singularities at the Lindbladt resonances and these points are at the same time turning points of the system, but global mathematical analysis in the region containing all singular points and turning points is very difficult. Thus we limit our consideration to the region $\Pi$ in this paper. The wave mechanism around the Lindbladt resonances $r_{I L R}$ and $r_{O L R}$ which are not contained in $\Pi$ is very important, and was studied locally by Mark [8] by the steller dynamical approach.

Now we prove the existence of solutions of the integral equation (4.10), which are of the order $O(\varepsilon)$, in several subregions of $\Pi$. First of all, let us describe the Stokes curves in $\Pi$ :

$$
\operatorname{Re} \int_{r_{0}}^{r} a_{0}(r) d r=0
$$

where $r_{0}$ is $r_{c e}$ or $r_{c o}$. Then from the assumption on $a_{0}^{2}(r)$, we have a Stokes curve configuration in the $I I$ as shown in Fig. 2. Here and in the following, $\operatorname{Re} f$ denotes the real part of $f$, and also $\operatorname{Im} f$ means the imaginary part of $f$.

The symbols $S$, in the figure denote subregions of $\Pi$ that are bounded by Stokes curves and the boundary of $\Pi$, and $L$, are some of the Stokes curves. By using the notation in the Fig. 2, we define four subregions $\hat{D}_{k}(k=1,2,3,4)$ of $\Pi$ containing parts of the real axis:

$$
\begin{array}{ll}
\hat{D}_{1}=\left\{S_{1} \cup L_{1} \cup S_{2}\right\} \cap \Pi, \quad \hat{D}_{2}=\hat{D}_{3}=\left\{S_{2} \cup L_{2} \cup S_{6} \cup L_{3} \cup S_{5}\right\} \cap \Pi, \\
\hat{D}_{4}=\left\{S_{3} \cup L_{4} \cup S_{4}\right\} \cap \Pi .
\end{array}
$$

To each $\hat{D}_{k}$, we assign two points $r_{k}, r_{k}^{\prime}$ and a Stokes curve $L_{k}$, and denote these quadruplets by $\left\{\hat{D}_{k}, r_{k}, r_{k}^{\prime}, L_{k}\right\} \quad(k=1,2,3,4)$ such as

$$
\begin{aligned}
& \left\{\hat{D}_{1}, r_{c e}, r_{c e}, L_{1}\right\}, \quad\left\{\hat{D}_{2}, r_{c e}, r_{c e}, L_{2}\right\}, \quad\left\{\hat{D}_{3}, r_{c o}, r_{3}^{\prime}, L_{3}\right\}, \\
& \left\{\hat{D}_{4}, r_{c o}, r_{4}^{\prime}, L_{4}\right\},
\end{aligned}
$$






Fig. 2. Stokes curve configuration of $a_{0}^{2}(r)$

where $r_{k}^{\prime}$ is some point in $\hat{D}_{k}$ respectively different from $r_{c o}$. By these we characterize the integrals of $h_{j}(r, \varepsilon)(\jmath=1,2,3)$ such that

$$
\begin{aligned}
& \int_{C_{1}}^{r} h_{1}(r, \varepsilon) d r=\int_{r_{i}}^{r} a_{0} d r+\int_{r_{\iota}^{\prime}}^{r} \varepsilon h_{11}^{(1)} d r, \\
& \int_{C_{2}}^{r} h_{2}(r, \varepsilon) d r=-\int_{r_{1}}^{r} a_{0} d r+\int_{r_{\imath}^{\prime}}^{r} \varepsilon h_{11}^{(2)} d r, \\
& \int_{C_{3}}^{r} h_{3}(r, \varepsilon) d r=\int_{r_{c e}}^{r}\left\{d_{0}+\varepsilon g_{3}\right\} d r,
\end{aligned}
$$

for $r \in \hat{D}_{k}(k=1,2,3,4)$. The branch $a_{0}$ and $\int^{r} a_{0} d r$ are determined by

$$
\operatorname{Re} \int_{r_{k}}^{r} a_{0}(r) d r>0 \quad \text { on } \quad L_{k}(k=1,2,3,4) .
$$

Thus we have defined the matrices $\Lambda(r, \varepsilon)$ that are characterized by $\left\{\hat{D}_{k}, r_{k}\right.$, $\left.r_{k}^{\prime}, L_{k}\right\}$ as above. Note that the integral for $h_{3}(r, \varepsilon)$ is the same for every $\hat{D}_{k}$.

By the suitable deformation of the above regions $\hat{D}_{k}$, we construct admissible regions $D_{k}(\gamma)$ where we can prove the existence of asymptotic solutions of (4.11). To do so, we introduce here a mapping $\xi=\xi\left(r, r_{0}\right)$ from the complex $r$-plane into the complex $\xi$-plane by

$$
\xi\left(r, r_{0}\right)=\int_{r_{0}}^{r} a_{0}(r) d r
$$


where $r_{0}$ may be any point of $\Pi$, but it is the most convenient to take it $r_{k}$ for $r_{0}$ if we consider the image of $\hat{D}_{k}$. Let us denote the image of $\hat{D}_{k}$ by $\hat{\mathscr{D}}_{k}$. Then $\hat{\mathscr{D}}_{k}$ is a bounded region in $\xi$-plane with vertical cuts. Next, we introduce the domains of influence $N_{c e}$ and $N_{c o}$, which are direct neighborhoods of $r_{c e}$ and $r_{c o}$ respectively, by

$$
\begin{aligned}
& N_{c e}=\left\{(r, \varepsilon):\left|r-r_{c e}\right|<N \varepsilon^{1 / 2}, 0<\varepsilon<\varepsilon_{0}\right\}, \\
& N_{c o}=\left\{(r, \varepsilon):\left|r-r_{c o}\right|<N \varepsilon^{1 / 5}, 0<\varepsilon<\varepsilon_{0}\right\},
\end{aligned}
$$

where $N$ and $\varepsilon_{0}$ are some positive constants. By taking $N$ sufficiently large and $\varepsilon_{0}$ sufficiently small, the condition (4.4) is satisfied and the series of $\varepsilon^{2} H_{2}(r, \varepsilon)$ is convergent for $r \in \Pi-N_{c e}-N_{c o}$ and $\varepsilon$ small enough. Let $\hat{D}$ be one of the regions $\hat{\mathscr{D}}_{k}(k=1,2,3,4)$ and let $\boldsymbol{\Omega}_{c e}$ and $\boldsymbol{\Omega}_{c o}$ be the images of $N_{c e}$ and $N_{c o}$ under the mapping (4.12), and consider regions $\hat{\mathscr{D}}-\mathscr{I}_{c e}-\Re_{c o} \equiv \hat{\mathscr{D}}[\varepsilon]$ in the $\xi$-plane. Now we change the region $\hat{\mathscr{D}}[\varepsilon]$ into $\mathscr{D}[\gamma, \varepsilon]$ for small positive $\gamma$ by deleting small neighborhoods of cuts and some portions near the boundary, so that it satisfies following conditions: For each $\imath, \jmath(\imath, \jmath=1,2,3)$, there exists a fixed point $\eta_{\imath \jmath}$ in $\mathscr{D}[\gamma, \varepsilon]$ and for every point $\xi$ in $\mathscr{D}[\gamma, \varepsilon]$ we can describe piecewise smooth curves $\mathcal{C}_{\imath \jmath}(s, \xi, \eta)$ for $0 \leqq s \leqq s_{0}(\xi)$ connecting $\xi$ and $\eta_{\imath \jmath}$, where $s$ denotes the arc length of the curve from $\xi$ and it satisfies

(1) $\mathcal{C}_{\imath j}\left(s, \xi, \eta_{\imath j}\right)$ is contained in $\mathscr{D}[\gamma, \varepsilon]$ and

$$
\mathcal{C}_{\imath j}\left(0, \xi, \eta_{\imath \jmath}\right)=\xi, \quad \mathcal{C}_{\imath \jmath}\left(s_{0}, \xi, \eta_{\imath \jmath}\right)=\eta_{\imath \jmath},
$$

(2) On this crrve, the following inequality is satisfied

$$
\frac{d \operatorname{Re} \gamma_{2}(r, \varepsilon)}{d s} \leqq 0
$$

where $\gamma_{\imath j}(r, \varepsilon)$ is defined in (4.11) and we consider here $\gamma_{\imath j}(r, \varepsilon)$ as a function of $\xi$ under the mapping (4.12).

We take the inverse image of this region $\mathscr{D}[\gamma, \varepsilon]$ and $\mathcal{C}_{\imath \jmath}\left(s, \xi, \eta_{\imath \jmath}\right)$ as the admissible region $D[\gamma]$ and paths of integral $C_{\imath}$, respectively. The union of all such admissible regions can cover a neighborhood of the real axis between $r_{c e}$ and $r_{c o}$ except domains of influence $N_{c e}$ and $N_{c o}$.

Now we specify how to construct the region $\mathscr{D}[\gamma, \varepsilon]$ and curves $\mathcal{C}_{2 \jmath}\left(s, \xi, \eta_{\imath_{\jmath}}\right)$. Firstly for $i=\jmath, \mathcal{C}_{\imath j}\left(s, \xi, \eta_{\imath \jmath}\right)$ can be any of the $\mathcal{C}_{\imath \jmath}\left(s, \xi, \eta_{\imath \jmath}\right)(\imath \neq j)$ specified below since $\gamma_{\imath j}(r, \varepsilon)=0$ for $\imath=\jmath$.

Let $\imath \neq \jmath$, and note that

$$
\begin{aligned}
\frac{d}{d s} \operatorname{Re} \gamma_{\imath j}(r, \varepsilon) & =\operatorname{Re} \frac{d}{d s} \gamma_{\imath j}(r, \varepsilon)=\operatorname{Re}\left(h_{\imath}(r, \varepsilon)-h_{j}(r, \varepsilon)\right) \frac{d r}{d s} \\
& =\operatorname{Re}\left\{\left(h_{\imath}(r, \varepsilon)-h_{\jmath}(r, \varepsilon)\right) a_{0}^{-1} \frac{d \xi}{d s}\right\} .
\end{aligned}
$$


Thus we have

$$
\begin{aligned}
& \frac{d}{d s} \operatorname{Re} \gamma_{12}(r, \varepsilon)=-\frac{d}{d s} \operatorname{Re} \gamma_{12}(r, \varepsilon)=\operatorname{Re}\left\{2+\varepsilon\left(h_{11}^{(1)}-h_{22}^{(1)}\right) a_{0}^{-1}\right\} \frac{d \xi}{d s} \\
& \frac{d}{d s} \operatorname{Re} \gamma_{13}(r, \varepsilon)=-\frac{d}{d s} \operatorname{Re} \gamma_{31}(r, \varepsilon)=\operatorname{Re}\left\{1-\frac{d_{0}}{a_{0}}+\varepsilon\left(h_{11}^{(1)}-g_{3}\right) a_{0}^{-1}\right\} \frac{d \xi}{d s} \\
& \frac{d}{d s} \operatorname{Re} \gamma_{23}(r, \varepsilon)=-\frac{d}{d s} \operatorname{Re} \gamma_{32}(r, \varepsilon)=\operatorname{Re}\left\{-1-\frac{d_{0}}{a_{0}}+\varepsilon\left(h_{22}^{(1)}-g_{3}\right) a_{0}^{-1}\right\} \frac{d \xi}{d s}
\end{aligned}
$$

The domains of influence of the turning points $r_{c e}$ and $r_{c o}$ are deleted from each $\hat{\mathscr{D}}[\varepsilon]$, so it is sufficient to construct $\mathcal{C}_{\imath j}\left(s, \xi, \eta_{i j}\right)$ satisfying (4.13), and instead of (4.14) the followings

$$
\begin{aligned}
& \operatorname{Re} \frac{d \xi}{d s}<-\gamma \quad \text { on } \mathcal{C}_{12}, \quad \operatorname{Re} \frac{d \xi}{d s}>\gamma \quad \text { on } \mathcal{C}_{21}, \\
& \operatorname{Re}\left(1-\frac{d_{0}}{a_{0}}\right) \frac{d \xi}{d s}<-\gamma \quad \text { on } \quad \mathcal{C}_{13}, \quad \operatorname{Re}\left(1-\frac{d_{0}}{a_{0}}\right) \frac{d \xi}{d s}>\gamma \quad \text { on } \quad \mathcal{C}_{31} \\
& \operatorname{Re}\left(-1-\frac{d_{0}}{a_{0}}\right) \frac{d \xi}{d s}<-\gamma \quad \text { on } \quad \mathcal{C}_{23}, \quad \operatorname{Re}\left(-1-\frac{d_{0}}{a_{0}}\right) \frac{d \xi}{d s}>\gamma \quad \text { on } \quad \mathcal{C}_{32},
\end{aligned}
$$

for a small positive constant $\gamma$, since the condition (4.14) can be satisfied by taking $\varepsilon$ sufficiently small. We show these for $\left\{\hat{D}_{1}, r_{c e}, r_{c e}, L_{1}\right\}$ and $\left\{\hat{D}_{2}, r_{c e}, r_{c e}, L_{1}\right\}$, for examples, and we can treat the other cases in a similar way.

(1) Case of $\left\{\hat{D}_{1}, r_{c e}, r_{c e}, L_{1}\right\}$.

Let $\hat{\mathscr{D}}_{1}, \tilde{r}_{c e}, \widetilde{L}_{1}$ be images of $\hat{D}_{1}, r_{c e}, L_{1}$ under the mapping $\xi=\xi\left(r, r_{c e}\right)$. $\tilde{r}_{c e}$ is the origin in the $\xi$-plane, $\widetilde{L}_{1}$ directs upwards and $\widetilde{L}_{2}, \widetilde{L}_{7}$ make a downward cut of $\hat{\mathscr{D}}_{1}$ (Fig. 3). From the definition (3.2) of the functions $a_{0}^{2}(r)$ and $d_{0}(r)$ and the way of determination of the branch $\int^{r} a_{0}(r) d r$ (and then $a_{0}(r)$ ), we have

$$
\frac{d_{0}(r)}{a_{0}(r)}=\frac{-k_{3}}{\imath k_{1}}=\frac{\Sigma}{2 k_{1}}
$$

where $k_{1}(r)>0$ for real $r>r_{c e}$ and $\Sigma$ positive for real $r>0$. Here we assume for simplicity that $\Sigma$ is a positive constant and $k_{1}=\alpha\left(r-r_{c e}\right)^{1 / 2}(\alpha$; positive constant) in $\hat{D}_{1}$.

The case in which the above assumptions do not satisfied could be handled with a slight modifications by taking $\hat{D}_{1}$ small.

Thus we have

$$
\xi\left(r, r_{c e}\right)=\int_{r_{c e}}^{r} i \alpha \lambda^{-1} \sqrt{r-r_{c e}} d r=\frac{2}{3} i \alpha \lambda^{-1}\left(r-r_{c e}\right)^{3 / 2},
$$


and then

$$
\begin{aligned}
& \left(r-r_{c e}\right)^{1 / 2}=\left(\frac{3 \lambda}{2 \alpha \imath} \xi\right)^{1 / 3}, \\
& \frac{d_{0}(r)}{a_{0}(r)}=-\frac{\Sigma}{2 k_{1}}=\frac{\Sigma}{2 \alpha\left(r-r_{c e}\right)^{1 / 2}}=\frac{\Sigma}{(2 \alpha)^{2 / 3}(3 \lambda)^{1 / 3}} e^{\frac{\pi}{6}} \xi^{-1 / 3} .
\end{aligned}
$$

It is easy to see that for $\xi \in \hat{\mathscr{D}}_{1}$

$$
\begin{aligned}
& \operatorname{Re} \frac{d_{0}(r)}{a_{0}(r)}>0 \text { if } \quad-\frac{\pi}{2}<\arg \xi<\frac{3 \pi}{2}, \\
&>0 \text { if } \quad-\frac{\pi}{2}<\arg \xi<\frac{\pi}{2}, \\
& \operatorname{Im} \frac{d_{0}(r)}{a_{0}(r)} \\
&<0 \quad \text { if } \quad \frac{\pi}{2}<\arg \xi<\frac{3 \pi}{2} .
\end{aligned}
$$

By assuming $\hat{D}_{1}$ is so small that for $\xi$ in $\hat{\mathscr{D}}_{1}$ we have

$$
\pm 1-\operatorname{Re}-\frac{d_{0}}{a_{0}} \leqq-\delta<0
$$

On the other hand, since we have

$$
\operatorname{Re}\left\{\left( \pm 1-\frac{d_{0}}{a_{0}}\right) \frac{d \xi}{d s}\right\}=\left( \pm 1-\operatorname{Re} \frac{d_{0}}{a_{0}}\right) \operatorname{Re} \frac{d \xi}{d s}+\operatorname{Im} \frac{d_{0}}{a_{0}} \operatorname{Im} \frac{d \xi}{d s}
$$

then it is clear how to construct the region $\mathscr{D}_{1}[\gamma, \varepsilon]$ and $\mathcal{C}_{\imath j}\left(s, \xi, \eta_{2 j}\right)(\imath, j=$ $1,2,3)$. For example, $\mathcal{C}_{13}\left(s, \xi, \eta_{13}\right)$ may consist of three segments which satisfy $\operatorname{Re} \frac{d \xi}{d s}>\frac{\gamma}{\delta}$, and $\operatorname{Im} \frac{d \xi}{d s}>0$ for the left side of $\widetilde{L}_{1} \cup \widetilde{L}_{2}$ and $\operatorname{Im} \frac{d \xi}{d s}<0$ for the other side (Fig. 3).

(2) Case of $\left\{\hat{D}_{2}, r_{c e}, r_{c e}, L_{2}\right\}$.

In this case, we remark that from the definition $a_{0}^{2}(r)$ and $d_{0}(r)$, and from the choice of the branch of $a_{0}(r)$ in $\hat{D}_{2}$,

$$
\frac{d_{0}}{a_{0}}=-\frac{1}{\sqrt{1-\left(1-\nu^{2}\right) Q^{2}}} .
$$

For real $\nu$ (that is, for real $\omega$ ), $0<1-\left(1-\nu^{2}\right) Q^{2}<1$ in $r_{c e}<r<r_{c o}$.

Then we can assume that in the region $\hat{\mathscr{D}}_{2}$

$$
\pm 1-\operatorname{Re} \frac{d_{0}}{a_{0}}<0
$$




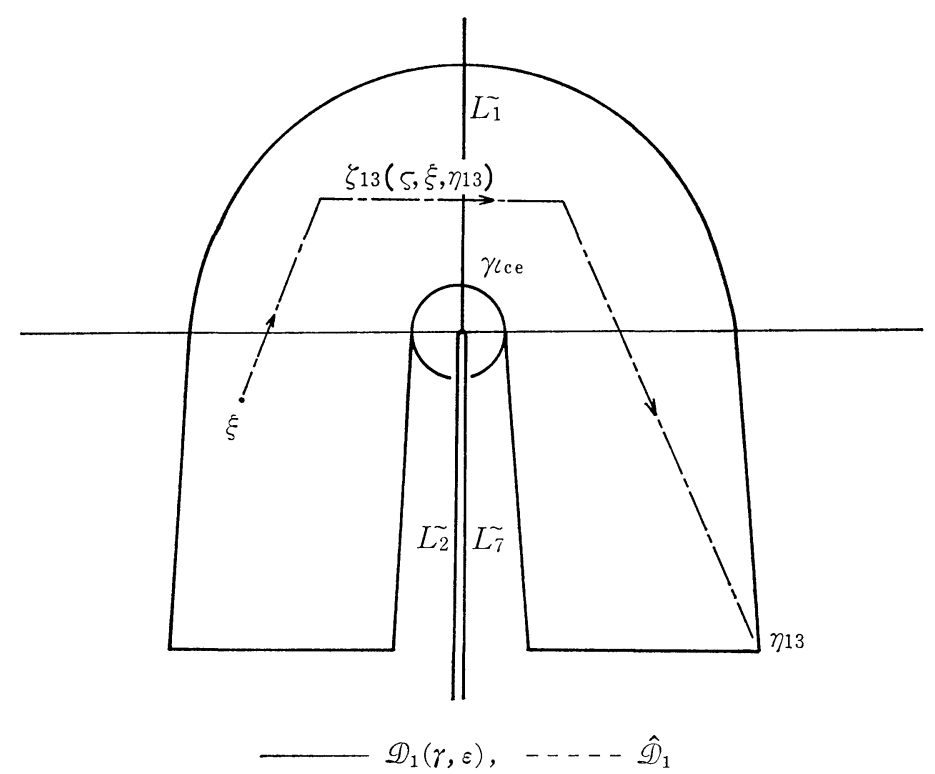

Fig. 3.

For $\operatorname{Im} \frac{d_{0}}{a_{0}}$, it is easy to see that $\operatorname{Im} d_{0} / a_{0}$ is positive (negative) for $\xi$ in the right side (left side) of $\widetilde{L}_{2}$. Therefore the construction of $\mathscr{D}_{2}[\gamma, \varepsilon]$ and $\mathcal{C}_{\imath \jmath}$ is almost straight forwards as in the case (1), Fig. 4. Qualitative figure of $D_{1}[\gamma]$ and $D_{2}[\gamma]$ in the $r$-plane is given in Fig. 5 .

Now we have specified so far admissible regions $D_{k}[\gamma](k=1,2,3,4)$ and the integral paths $C_{\imath \jmath}(i, \jmath=1,2,3)$ in (4.11), from which it is easy to prove the existence of solutions of the integral equation (4.11) and of the differential equation (4.8), (4.9) in each admissible region $D_{k}[\gamma]$ by the standard application of the successive approximations, and also it is clear that these solutions are of the order $O(\varepsilon)$.

By transforming the differential equation (4.8) back to the differential equation (3.2) through the transformations (4.7), (4.3), (4.1) and (3.3), we have thus established the following existence theorem.

THEOREM 4.1. The differential system (3.2) has a fundamental system of solutions such that it satısfies in each admissible region $D_{k}[\gamma](k=1,2,3,4)$

$$
U^{(k)}(r, \varepsilon)=\left(P_{0}+\varepsilon P_{1}\right) T_{0}\left(E+\varepsilon T_{1}\right)(E+Z) \exp \Lambda(r, \varepsilon),
$$

where the matrices $P_{0}$ and $P_{1}$ are defined in (3.3), $T_{0}$ in (4.1), $T_{1}$ in (4.3), $\Lambda(r, \varepsilon)$ in (4.6) respectively, and the remander term $Z(r, \varepsilon)$ is of the order $O(\varepsilon)$. The expression (4.16) can be rewritten as 


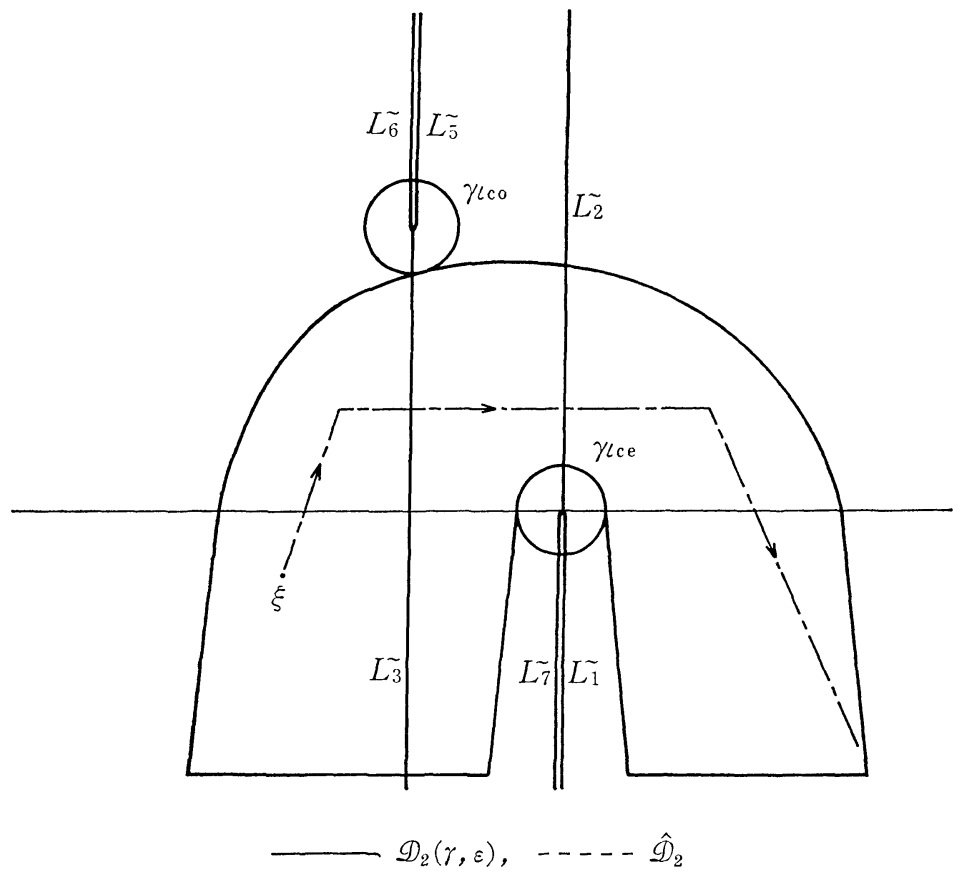

Fig. 4.

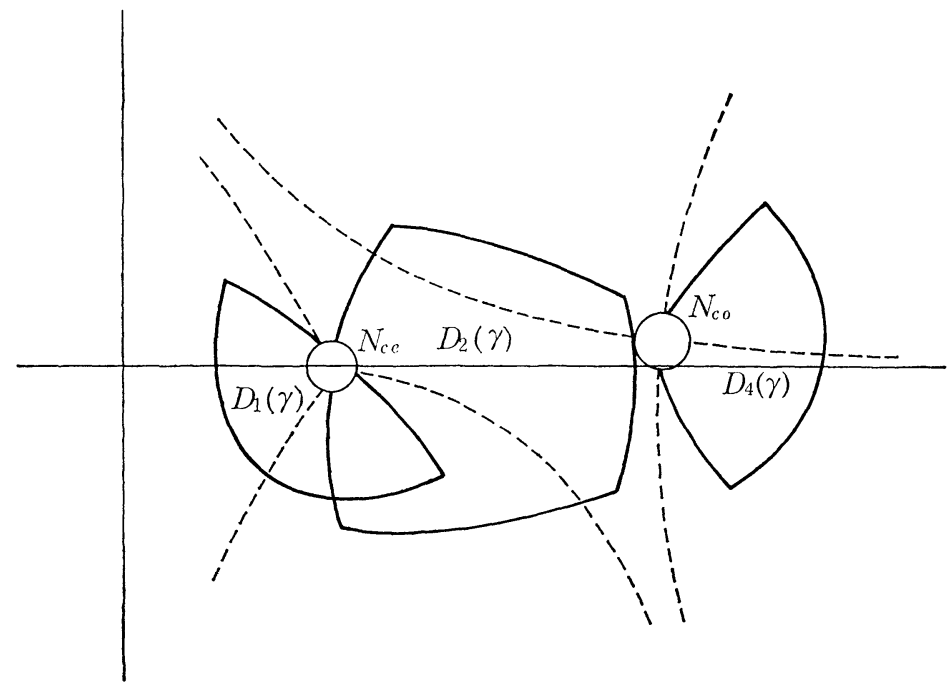

Fig. 5. Admissible regions $D_{1}[\gamma]$ and $D_{3}\left[\gamma_{-}^{-}\right.$ 


$$
\begin{aligned}
U^{(k)}(r, \varepsilon)= & \left\{\left(\begin{array}{ccc}
1 & -1 & 0 \\
a_{0} & a_{0} & 0 \\
\frac{c_{0}}{a_{0}-d_{0}} & \frac{c_{0}}{a_{0}+d_{0}} & 1
\end{array}\right)(E+\tilde{Z}(r, \varepsilon))\right\} \\
& \cdot \exp \left(\begin{array}{ccc}
\int^{r} \frac{h_{1}(r, \varepsilon)}{\varepsilon} d r, & 0, \\
0, & \int^{r} \frac{h_{2}(r, \varepsilon)}{\varepsilon} d r, & 0 \\
0, & 0, & \int^{r} \frac{h_{3}(r, \varepsilon)}{\varepsilon} d r
\end{array}\right),
\end{aligned}
$$

where $h_{\imath}(r, \varepsilon)(\imath=1,2,3)$ are defined by $(4.10)$, and $\tilde{Z}(r, \varepsilon)=O(\varepsilon)$.

The following corollaries are obvious;

COROLlary 4.1. The differentral system (3.5) has a fundamental system of solutions $\widetilde{W}^{(k)}(r, \varepsilon)$ such that in each admissible region $D_{k}[\gamma](k=1,2,3,4)$ it has an asymptotic expansion of the form

$$
\widetilde{W}^{(k)}(r, \varepsilon)=\left(\begin{array}{ccc}
1 & -1 & 0 \\
a_{0} & a_{0} & 0 \\
0 & 0 & 1
\end{array}\right)(E+\tilde{Z}(r, \varepsilon)) \exp \Lambda(r, \varepsilon),
$$

where $\tilde{Z}(r, \varepsilon)=O(\varepsilon)$, and $\Lambda(r, \varepsilon)$ is the same as in (4.17).

COROLlary 4.2. There exists a fundamental system of solutions $W^{(k)}(r, \varepsilon)$ of the differential system (3.4) whrch has the same asymptotic expansion as (4.18), and satısfies in each admissible region $D_{k}[\gamma](k=1,2,3,4)$ that

$$
\left(\begin{array}{ccc}
1 & -1 & 0 \\
a_{0} & a_{0} & 0 \\
0 & 0 & 1
\end{array}\right)^{-1}\left\{W^{(k)}(r, \varepsilon)-\widetilde{W}^{(k)}(r, \varepsilon)\right\} \exp \{-\Lambda(r, \varepsilon)\}=O(\varepsilon)
$$

\section{§5. Splitting property and dispersion relation.}

We have established in the previous section that there exists a fundamental system of solutions of the differential system (3.3) with asymptotic property (4.17) in each admissible region. Each of the solutions carries the exponential function, $\exp \Lambda_{j}(r, \varepsilon)(\jmath=1,2,3)$, and so let us call all solutions carrying $\exp \Lambda_{\jmath}$ the $\exp \Lambda_{\jmath}$ type solutions. We shall show in this section a splitting property which means that the $\exp \Lambda_{3}$ type solution is asymptotically independent from other two type solutions throughout the region $\Pi$ and conversely $\exp \Lambda_{1}$ and $\exp \Lambda_{2}$ type solutions 
are independent from the exp $\Lambda_{3}$ type solution, if the differential system (3.2) does not have singularity (pole) at the corotation point $r=r_{c o}$. The singularity of the system (3.2) at $r=r_{c o}$ comes from the term

$$
\frac{2 m \Omega}{r^{2} \kappa \nu} \frac{d \log \left(\kappa^{2} / \sigma_{0} \Omega\right)}{d \log r}
$$

in the system (3.1), but we can avoid the singularity by replacing this term with a regular term which is obtained from the steller dynamical approach instead of the hydrodynamical approach, Lau and Bertin [3]. Nevertheless, further mathematical studies around the singularity is important.

At first, it is proved that the splitting property is valid over $D_{1}[\gamma] \cup D_{2}[\gamma]$, that is, the fundamental system $U^{(1)}$ defined by the asymptotic expansion (4.17) in $D_{1}[\gamma]$ is expressed in $D_{2}[\gamma]$ by $U^{(2)}$ in the splitting way. Let $D_{5}[\gamma]$ be an auxiliary admissible region which is obtained from $\hat{D}_{5}$ :

$$
\hat{D}_{5}=\left\{S_{1} \cup L_{7} \cup S_{6} \cup L_{3} \cup S_{5}\right\} \cap \Pi,
$$

and $U^{(5)}$ be a corresponding fundamental system of solutions defined by the same method as in the previous section. We denote three linearly independent solutions of $U^{(i)}$ by $\left\{u_{1}^{(i)}, u_{2}^{(i)}, u_{3}^{(i)}\right\},(\imath=1,2,5)$, where $u_{k}^{(i)}$ corresponds to $\exp \Lambda_{k}$ type solution $(k=1,2,3)$. Now since the point $r_{c e}$ is a regular point of the equation, the conncetion formulas between them can be expressed as

$$
\begin{aligned}
& u_{1}^{(2)}=a_{11} u_{1}^{(1)}+a_{12} u_{2}^{(1)}+a_{13} u_{3}^{(1)} \\
& u_{2}^{(2)}=a_{21} u_{1}^{(1)}+a_{22} u_{2}^{(1)}+a_{23} u_{3}^{(1)}, \\
& u_{3}^{(2)}=a_{31} u_{1}^{(1)}+a_{32} u_{2}^{(1)}+a_{33} u_{3}^{(1)} \\
& u_{1}^{(5)}=b_{11} u_{1}^{(2)}+b_{12} u_{2}^{(2)}+b_{13} u_{3}^{(2)} \\
& u_{2}^{(5)}=b_{21} u_{1}^{(2)}+b_{22} u_{2}^{(2)}+b_{23} u_{3}^{(2)}, \\
& u_{3}^{(5)}=b_{31} u_{1}^{(2)}+b_{32} u_{2}^{(2)}+b_{33} u_{3}^{(2)} \\
& u_{1}^{(1)}=c_{11} u_{1}^{(5)}+c_{12} u_{2}^{(5)}+c_{13} u_{3}^{(5)} \\
& u_{2}^{(1)}=c_{21} u_{1}^{(5)}+c_{22} u_{2}^{(5)}+c_{23} u_{3}^{(5)} . \\
& u_{3}^{(1)}=c_{31} u_{1}^{(5)}+c_{32} u_{2}^{(5)}+c_{33} u_{3}^{(5)}
\end{aligned}
$$

As in the previous section, we have in the neighborhood of $r_{c e}$

$$
\begin{aligned}
\operatorname{Re} \int_{r_{c e}}^{r}\left( \pm a_{0}-d_{0}\right) d r & =\operatorname{Re}\left\{\int_{r_{c e}}^{r} \pm \imath \alpha \lambda^{-1} \sqrt{r-r_{c e}} d r-\int_{r_{c e}}^{r} i \lambda^{-1} \frac{\Sigma}{2} d r\right\} \\
& =\operatorname{Im} \lambda^{-1}\left(r-r_{c e}\right)\left( \pm \frac{2}{3} \alpha \sqrt{r-r_{c e}}-\frac{\Sigma}{2}\right)
\end{aligned}
$$


where $\lambda$ is a positive parameter, $\alpha$ and $\Sigma$ are assumed to be positive constant, and the $\operatorname{sign}( \pm)$ is determined by the branch of $a_{0}$ in the admissible regions. Therefore for $r$ close to $r_{c e}$, we have

$$
\operatorname{Re}\left\{\int_{r_{c e}}^{r} \pm a_{0} d r-\int_{r_{c e}}^{r} d_{0} d r\right\} \stackrel{\doteq}{ } \operatorname{Im}\left[\frac{\Sigma}{2} \lambda^{-1}\left(r-r_{c e}\right)\right]\left\{\begin{array}{l}
<0 \text { if } \operatorname{Im}\left(r-r_{c e}\right)>0 \\
>0 \text { if } \operatorname{Im}\left(r-r_{c e}\right)<0
\end{array} .\right.
$$

This means that the region $D_{1}[\gamma] \cap D_{2}[\gamma]$ contains a region where

$$
\operatorname{Re} \int_{r_{c e}}^{r} a_{0} d r>\operatorname{Re} \int_{r_{c e}}^{r} d_{0} d r
$$

and from this fact we can see that the coefficients $a_{31}$ and $a_{32}$ in (5.1a) asymptotically vanish. Then we have proved that the $\exp \Lambda_{3}$ type solution $u_{3}^{(1)}$ is also the same type solution in $D_{2}[\gamma]$. By the analogous reasons we can easily conclude that $b_{13} \cong b_{23} \cong b_{22} \cong 0$ in (5.1b) and $c_{13} \cong c_{22} \cong c_{23} \cong c_{31} \cong c_{32} \cong 0$ in (5.1c), which means that $u_{1}^{(1)}$ and $u_{2}^{(1)}$ are linear combinations of $u_{1}^{(2)}$ and $u_{2}^{(2)}$. Thus we have obtained the splitting property over $D_{1}[\gamma] \cup D_{2}[\gamma]$. The splitting property over $D_{2}[\gamma]$ $\cup D_{3}[\gamma]$ is trivial, and for $D_{3}[\gamma] \cup D_{4}[\gamma]$ it is easily proved if $r_{c o}$ is not a singular point by the analogous method as above.

THEOREM 5.1. The fundamental systems of solutions of (3.2) characterized by the asymptotic expansions (4.17) have the splitting property throughout the region $\Pi$ if $r=r_{\text {co }}$ is a regular point of the differential system (3.2).

The splitting property for the fundamental systems of solutions of (3.5) is defined analogously and is valid from the outset, and that for the system (3.4) is also correct because the solutions of (3.2) are obtained from those of (3.4) by applying the transformation (3.3). It is clear from the method of calculation of connection formulas around turning points, Evgrafov and Fedoryuk [2], the leading terms of connection matrices between two fundamental systems of the differential system (3.4) are identical to those of the differential system (3.5).

This fact and the splitting property give us the following main theorem.

THEOREM 5.2. Suppose that the differential systems (3.4) and (3.5) have no singular point in $\Pi$ by replacing singular terms with regular terms. Then, the principal terms of asymptotic expansions of the fundamental systems of solutions of (3.4) are identical to those of (3.5) throughout II. If we consider the boundary value problem subject to the boundary conditions (3.7), then the leading terms of global dispersion relations of (3.4) and (3.5), if exist, have the same forms, and in particular, they are identical to that of the second order ordinary differential equation (3.6).

Thus the problem of finding the global dispersion relation for the boundary value problem of the differential system (3.4) has been reduced to that of the second order ordinary differential equation (3.6). To make use of the results for 
the second order equation with a coefficient of a cubic polynomial [9], it is convenient to rewrite the equation (3.6) slightly. Since $g_{2}(r)$ is assumed to be regular at $r=r_{c o}$, we can write the function $a_{0}^{2}(r)+g_{2}(r) \varepsilon$ in the neighborhood of $r_{c o}$ as follows :

$$
a_{0}^{2}(r)+\varepsilon g_{2}(r)=-a_{00}\left\{\left(r-r_{c o}\right)^{2}+\varepsilon J^{2}+0\left(\left(r-r_{c o}\right)^{3}\right)+0\left(\varepsilon\left(r-r_{c o}\right)\right)\right\},
$$

where $a_{00}$ and $J^{2}$ are positive constants, and $g_{2}\left(r_{c 0}\right)=a_{00} J^{2}$. That $a_{00} J^{2}>0$ corresponds to the so-called Jean's instability. We put

then

$$
\begin{aligned}
& \tilde{a}_{0}^{2}(r)=a_{0}^{2}(r)+a_{00} p^{2} \quad\left(p^{2}=\varepsilon J^{2}\right), \\
& \tilde{g}_{2}(r)=g_{2}(r)-a_{00} J^{2} \\
& a_{0}^{2}(r)+\varepsilon_{g_{2}}(r)=\tilde{a}_{v}^{2}(r)+\varepsilon \tilde{g}_{2}(r) .
\end{aligned}
$$

Now let us consider the differential equation (3.6) with substitution $a_{\nu}^{2}(r)$ and $g_{2}(r)$ by $\tilde{a}_{0}^{2}(r)$ and $\tilde{g}_{2}(r)$. Then there are three simple turning points $\tilde{r}_{c e}, r_{c o} \pm \tilde{p}_{2}$ where $\tilde{r}_{c e}$ is close to $r_{c e}$ and $r_{c o} \pm \tilde{p}_{2}$ is close to $r_{c o}$. It is clear that we can construct fundamental systems of solutions of (3.6) in several admissible regions by the similar method as in $\S 4$.

We assume here that the group velocity is positive for $r>r_{c o}$ or

$$
\operatorname{Re}\left\{\imath \frac{\partial \tilde{a}_{n}}{\partial \omega}\right\}^{-1}>\rho \quad \text { for } \quad r>r_{c o} \div \delta
$$

where $\rho$ and $\delta$ are positive constants.

Under these assumptions, we can follow the procedure in [9] and obtain

THEOREM 5.3. The leading terms of global dispersion relation for the boundary value problem (3.6), (3.7) and (3.4), (3.7) have the form

$$
\exp \left[\frac{2}{\varepsilon} \int_{\tilde{r}_{c e}}^{r_{c o}-\tilde{p}_{2}}\left\{\tilde{a}_{0}(r)+\frac{\varepsilon \tilde{g}_{2}(r)}{2 \tilde{a}_{0}}\right\} d r\right]=-\frac{\Gamma\left(\frac{1}{2}+q_{2} / 2\right)}{\sqrt{ } 2 \pi}\left(\frac{q^{2}}{2}\right)^{-q^{2 / 2}} e^{q^{2} / 2}
$$

where $q^{2}=\imath \sqrt{a_{00}} J^{2}$.

Lastly we give here a few remarks

Remark 1. We have proved that the study of the third order differential system (3.1) or (3.2) is asymptotically reduced to that of the second order differential equation (3.6) which has the same principal term $a_{0}^{2}(x)$ as that of the equation (2.9). But to estimate influences of higher order terms of the equations, it is reasonable to study the equation (3.6).

Remark 2. In his previous paper [9], the author gave a dispersion relation for the second order differential equation of the form (2.9) as a conjecture (see 
[9], (5.1)). But we can consider that its validity has been established in this paper.

Remark 3. So far in the analysis of equations of the form (2.9), only two types of solutions $\exp \Lambda_{1}$ and $\exp \Lambda_{2}$ type solutions in our terminology have been considered. But in this note we have obtained the third type solution, $\exp \Lambda_{3}$ type solution, and so roles of this solution in the density wave theory must be studied. In the region $\Pi$, $\exp \Lambda_{3}$ type solution is independent from other types, but they may interact with each other at the neighborhood of the Lindbladt resonances $r_{I L R}$ and $r_{O L R}$.

\section{Appendix}

In this appendix, we construct in detail the block diagonalization (3.3). The letters $A, B, C, D, S$, and $T$ used here are irrelevant to same letters in the section $2,3,4$ and 5 .

Les us rewrite the equation (3.2) in the following form

$$
\varepsilon U^{\prime}=\left[\begin{array}{ll}
A & B \\
C & D
\end{array}\right] U, \quad U=\left(\begin{array}{l}
u_{1} \\
u_{2} \\
u_{3}
\end{array}\right),
$$

where

$$
A \equiv A_{0}+\varepsilon A_{1}=\left[\begin{array}{cc}
0 & 1 \\
a_{0}^{2} & 0
\end{array}\right]+\varepsilon\left[\begin{array}{cc}
0 & 0 \\
a_{1} & 0
\end{array}\right], \quad B \equiv \varepsilon B_{1}=\varepsilon\left[\begin{array}{c}
0 \\
b_{1}
\end{array}\right]
$$

$$
C \equiv C_{0}=\left[\begin{array}{cc}
c_{0}, & 0
\end{array}\right], \quad D \equiv D_{0}+\varepsilon D_{1}=d_{0}+\varepsilon d_{1} .
$$

Accordingly, we construct the transformation of the form

$$
U=\left[\begin{array}{cc}
E_{2}+\varepsilon S T & \varepsilon S \\
T & 1
\end{array}\right] W, \quad W=\left(\begin{array}{l}
w_{1} \\
w_{2} \\
w_{3}
\end{array}\right),
$$

where $E_{2}$ is the 2-dim unit matrix, and $S, T$ are (2-1), (1-2) matrix functions to be determined. By applying (A.3) to (A.1), we get

$$
\varepsilon W^{\prime}=G W, \quad G=\left[\begin{array}{ll}
G_{11} & G_{12} \\
G_{21} & G_{22}
\end{array}\right]
$$

with

$$
G_{11}=A\left(E_{2}+\varepsilon S T\right)+B T-\varepsilon S\left(C+\varepsilon C S T+D T+\varepsilon T^{\prime}\right),
$$




$$
\begin{aligned}
& G_{12}=\varepsilon A S+B+\varepsilon^{2} S^{\prime}-\varepsilon S\left\{\varepsilon C S+D-\varepsilon^{2}(T S)^{\prime}\right\}, \\
& G_{21}=-T(A+\varepsilon A S T+B T)+(1+\varepsilon T S)\left(C+\varepsilon C S T+D T+\varepsilon T^{\prime}\right), \\
& G_{22}=-T\left(\varepsilon A S+B+\varepsilon^{2} S^{\prime}\right)+(I+\varepsilon T S)\left\{\varepsilon C S+D-\varepsilon^{2}(T S)^{\prime}\right\} .
\end{aligned}
$$

Here we put $S=S_{0}$ and $T=T_{0}+\varepsilon T_{1}$ in the above equations and replace the matrices $A, B, C$ and $D$ by their power series of $\varepsilon(A .2)$. Then we have

$$
\begin{aligned}
& G_{11}=A_{0}+\varepsilon\left\{A_{1}-S_{0} C_{0}+\left(A_{0} S_{0}-S_{0} D_{0}+B_{1}\right) T_{0}\right\} \\
& +\varepsilon^{2}\left\{A_{1} S_{0} T_{0}-S_{0} C_{0} S_{0} T_{0}-S_{0} D_{1} T_{0}-S_{0} T_{0}^{\prime}+\left(A_{0} S_{0}-S_{0} D_{0}+B_{1}\right) T_{1}\right\} \\
& +\varepsilon^{3}\left\{A_{1} S_{0} T_{1}-S_{0} C_{0} S_{0} T_{1}-S_{0} D_{1} T_{1}-S_{0} T_{1}^{\prime}\right\}, \\
& G_{12}=\varepsilon\left(A_{0} S_{0}-S_{0} D_{0}+B_{1}\right)+\varepsilon^{2}\left(A_{1} S_{0}+S_{0}^{\prime}-S_{0} C_{0} S_{0}-S_{0} D_{1}\right) \\
& +\varepsilon^{3} S\left(T_{0} S_{0}\right)^{\prime}+\varepsilon^{4} S_{0}\left(T_{1} S_{0}\right)^{\prime}, \\
& G_{21}=-T_{0} A_{0}+D_{0} T_{0}+C_{0}+\varepsilon\left\{-T_{0} A_{1}-T_{1} A_{0}+C_{0} S_{0} T_{0}+D_{0} T_{1}+D_{1} T_{0}-T_{0}^{\prime}+T_{0} S_{0} C_{0}\right. \\
& \left.-T_{0}\left(A_{0} S_{0}-S_{0} D_{0}+B_{1}\right) T_{0}\right\}+\varepsilon^{2}\left\{-T_{1} A_{1}-T_{0} A_{1} S_{0} T_{0}+C_{0} S_{0} T_{1}+D_{1} T_{1}+T_{1}^{\prime}\right. \\
& +T_{0} S_{0}\left(C_{0} S_{0} T_{0}+D_{1} T_{0}+T_{0}^{\prime}\right)+T_{1} S_{0} C_{0}-T_{0}\left(A_{0} S_{0}-S_{0} D_{0}+B_{1}\right) T_{1} \\
& \left.-T_{1}\left(A_{0} S_{0}-S_{0} D_{0}+B_{1}\right) T_{0}\right\}+\varepsilon^{3}\left\{-T_{0} A_{1} S_{0} T_{1}-T_{1} A_{1} S_{0} T_{0}\right. \\
& +T_{0} S_{0}\left(C_{0} S_{0} T_{1}+D_{1} T_{1}+T_{1}^{\prime}\right)+T_{1} S_{0}\left(C_{0} S_{0} T_{0}+D_{1} T_{0}+T_{0}^{\prime}\right) \\
& \left.-T_{1}\left(A_{0} S_{0}-S_{0} D_{0}+B_{1}\right) T_{1}\right\}+\varepsilon^{4}\left\{-T_{1} A_{1} S_{0} T_{1}+T_{1} S_{0} C_{0} S_{0} T_{1}\right. \\
& \left.+T_{1} S_{0}\left(D_{1} T_{1}+T_{1}^{\prime}\right)\right\} \text {, } \\
& G_{22}=D_{0}+\varepsilon\left\{D_{1}+C_{0} S_{0}-T_{0}\left(A_{0} S_{0}-S_{0} D_{0}+B_{1}\right)\right\} \\
& +\varepsilon^{2}\left\{-T_{0}\left(A_{1} S_{0}+S_{0}^{\prime}-S_{0} C_{0} S_{0}-S_{0} D_{1}\right)-\left(T_{0} S_{0}\right)^{\prime}-T_{1}\left(A_{0} S_{0}-S_{0} D_{0}+B_{1}\right)\right\} \\
& +\varepsilon^{3}\left\{-T_{1}\left(A_{1} S_{0}+S_{0}^{\prime}-S_{0} C_{0} S_{0}-S_{0} D_{1}\right)-\left(T_{1} S_{0}\right)^{\prime}+T_{0} S_{0}\left(T_{0} S_{0}\right)^{\prime}\right\} \\
& +\varepsilon^{4}\left\{T_{1} S_{0}\left(T_{0} S_{0}\right)^{\prime}+T_{0} S_{0}\left(T_{1} S_{0}\right)^{\prime}\right\}+\varepsilon^{5} T_{1} S_{0}\left(T_{1} S_{0}\right)^{\prime} .
\end{aligned}
$$

In order that $G_{12}$ and $G_{21}$ are of the order $\varepsilon^{2}, S_{0}, T_{0}$ and $T_{1}$ must satisfy

$$
\begin{aligned}
& A_{0} S_{0}-S_{0} D_{0}+B_{1}=0, \\
- & T_{0} A_{0}+D_{0} T_{0}+C_{0}=0, \\
- & T_{1} A_{0}+D_{0} T_{1}-T_{0} A_{1}+C_{0} S_{0} T_{0}+D_{1} T_{0}+T_{0}^{\prime}+T_{0} S_{0} C_{0}=0 .
\end{aligned}
$$

Thus we have 


$$
\begin{gathered}
S_{0} \equiv\left(\begin{array}{c}
s_{1}^{(0)} \\
s_{2}^{(0)}
\end{array}\right)=\left(\begin{array}{c}
\frac{b_{1}}{d_{0}^{2}-a_{0}^{2}} \\
\frac{b_{1} d_{0}}{d_{0}^{2}-a_{0}^{2}}
\end{array}\right), \\
T_{0}=\left(t_{1}^{(0)}, t_{2}^{(0)}\right)=\left(\frac{c_{0} d_{0}}{a_{0}^{2}-d_{0}^{2}}, \frac{c_{0}}{a_{0}^{2}-d_{0}^{2}}\right), \\
T_{1}=\left(t_{1}^{(1)}, t_{2}^{(1)}\right)=\left(\frac{\alpha_{1} d_{0}+\alpha_{2}}{a_{0}^{2}-d_{0}^{2}}, \frac{\alpha_{1} d_{0}+\alpha_{2} a_{0}^{2}}{a_{0}^{2}-d_{0}^{2}}\right),
\end{gathered}
$$

where

$$
\begin{aligned}
& \alpha_{1}=-\frac{c_{0}\left(d_{0} d_{1}-a_{1}\right)}{a_{0}^{2}-d_{0}^{2}}-\frac{3 b_{1} c_{0}^{2} d_{0}}{\left(a_{0}^{2}-d_{0}^{2}\right)^{2}}+\left(\frac{c_{0} d_{0}}{a_{0}^{2}-d_{0}^{2}}\right)^{\prime}, \\
& \alpha_{2}=-\frac{c_{0} d_{1}}{a_{0}^{2}-d_{0}^{2}}-\frac{b_{1} c_{0}^{2}}{\left(a_{0}^{2}-d_{0}^{2}\right)^{2}}+\left(\frac{c_{0}}{a_{0}^{2}-d_{0}^{2}}\right)^{\prime} .
\end{aligned}
$$

By these determinations of $S$ and $T$, the transformation (A.3) becomes (A.5)

$$
\begin{gathered}
U=\left(P_{0}+\varepsilon P_{1}\right) W, \\
P_{0}=\left(\begin{array}{ccc}
1 & 0 & 0 \\
0 & 1 & 0 \\
\frac{c_{0} d_{0}}{a_{0}^{2}-d_{0}^{2}}-\frac{c_{0}}{a_{0}^{2}-d_{0}^{2}} & 1
\end{array}\right), \\
P_{1}=\left(\begin{array}{ccc}
\frac{-b_{1} c_{0} d_{0}}{\left(a_{0}^{2}-d_{0}^{2}\right)^{2}} & -\frac{-b_{1} c_{0}}{\left(a_{0}^{2}-d_{0}^{2}\right)^{2}} & \frac{-b_{1}}{a_{0}^{2}-d_{0}^{2}} \\
\frac{-b_{1} c_{0} d_{0}^{2}}{\left(a_{0}^{2}-d_{0}^{2}\right)^{2}} & \frac{-b_{1} c_{0} d_{0}}{\left(a_{0}^{2}-d_{0}^{2}\right)^{2}} & \frac{-b_{1} d_{0}}{a_{0}^{2}-d_{0}^{2}} \\
\frac{a_{0}^{2} \alpha_{2}+d_{0} \alpha_{1}}{a_{0}^{2}-d_{0}^{2}} & \frac{\alpha_{1}+d_{0} \alpha_{2}}{a_{0}^{2}-d_{0}^{2}} & 0
\end{array}\right),
\end{gathered}
$$

and the resultant equation is

$$
\varepsilon \frac{d W}{d r}=\left\{G_{0}(r)+\varepsilon G_{1}(r)+\varepsilon^{2} G_{2}(r, \varepsilon)\right\} W
$$

where

$$
G_{0}(r)=\left(\begin{array}{ccc}
0 & 1 & 0 \\
a_{0}^{2} & 0 & 0 \\
0 & 0 & d_{0}
\end{array}\right),
$$




$$
G_{1}(r)=\left(\begin{array}{ccc}
\frac{b_{1} c_{0}}{a_{0}^{2}-d_{0}^{2}} & 0 & 0 \\
a_{1}+\frac{b_{1} c_{0} d_{0}}{a_{0}^{2}-d_{0}^{2}} & 0 & 0 \\
0 & 0 & d_{1}-\frac{b_{1} c_{0}}{a_{0}^{2}-d_{0}^{2}}
\end{array}\right),
$$

and $G_{2}(r, \varepsilon)$ is a polynomial of $\varepsilon$ of degree 3 and its coefficients are rational functions of $a_{0}, a_{1}, b_{1}, c_{0}, d_{0}$ and $d_{1}$. Since $a_{0}^{2}-d_{0}^{2}=\kappa^{2}\left(1-\nu^{2}\right) / a^{2}$, it is easy to count the order of poles of $S_{0}, T_{0}$ and $T_{1}$ at each singularity. We give at Table 1 the order of poles of $A_{1}, B_{1}, D_{1}$ and $S_{0}, T_{0}, T_{1}$.

\begin{tabular}{|c|c|c|c|c|}
\hline & center $(r=0)$ & $r_{I L R}$ & $r_{c o}$ & $r_{O L R}$ \\
\hline$a_{1}$ & 2 & 2 & 1 & 2 \\
$b_{1}$ & 2 & 1 & 1 & 1 \\
$d_{1}$ & 2 & 1 & 0 & 1 \\
$A_{1}$ & 2 & 2 & 1 & 2 \\
$B_{1}$ & 2 & 1 & 1 & 1 \\
$D_{1}$ & 2 & 1 & 0 & 1 \\
$S_{0}$ & 2 & 2 & 1 & 2 \\
$T_{0}$ & 0 & 1 & 0 & 1 \\
$T_{1}$ & 2 & 4 & 1 & 4 \\
\hline
\end{tabular}

Table 1. Order of poles at singularities.

From the expression of $G_{\imath j}(r)(\imath, \jmath=1,2)$ in power series of $\varepsilon$ and the above Table, the remainder term $G_{2}(r, \varepsilon) \varepsilon^{2}$ can be written in the neighborhoods of singularities as

$$
G_{2}(r, \varepsilon) \varepsilon^{2}=\sum_{\imath=2}^{5} G_{\imath}^{(l)}(r)\left\{\tau^{(l)}(r) \varepsilon\right\}^{\imath}
$$

where $l$ denotes one of singularities $0, r_{I L R}, r_{c o}$ and $r_{O L R}, G_{\imath}^{(l)}(r)$ is bounded in the neighborhood of singular point $l$ and $\tau^{(l)}(r)$ is defined by

$$
\tau^{(l)}(r)=\left\{\begin{array}{cl}
r^{-2} & \text { for } l=0, \\
\left(r-r_{I L R}\right)^{-3} & \text { for } l=r_{I L R}, \\
\left(r-r_{c o}\right)^{-1} & \text { for } l=r_{c o}, \\
\left(r-r_{O L R}\right)^{-3} & \text { for } l=r_{O L R} .
\end{array}\right.
$$

Acknowledgements. The author wishes to thank Professors C.C. Lin and 
Y.Y. Lau for introducing him many interesting mathematical problems about galaxies during the period when he was visiting M.I.T.

He wishes also to thank Professor Y. Hirasawa and Dr, H. Yamada for many helpful discussions and valuable comments.

This work is supported by Grant-in-Aid for Scientific Research from the Ministry of Education. (No. 454025).

\section{REFERENCES}

[1] Bertin, G. AND J.W.K. MARK, Local density-potential relation for spiral density waves in galaxies. SIAM J. Appl. Math., 36 (1979) to appear.

[2] Evgrafov, M. A. And M. V. Fedoryuk, Asymptotic behavior as $\lambda \rightarrow \infty$ of the solution of the equation $w^{\prime \prime}(z)-p(z \cdot \lambda) w(z)=0$ in the complex $z$-plane. Russian Math. Surveys, 21 (1966), 1-48.

[3] LAU, Y.Y. AND G. Bertin, Discrete spiral modes, spiral waves, and the local dispersion relationship. Astrophys. J., 226 (1978), 508-520.

[4] LAU, Y.Y. AND J.W. K. MARK, Unstable spiral modes in disk shaped galaxies . Enhancement of growth rate. Proc. Natl. Acad. Sci. U.S.A., 73 (1976), 3785 $-3787$.

[5] Lin,C.C. AND Y.Y. LAU, On sprral waves in galxies-A gas dynamical approach. SIAM J. Appl. Math., 29 (1975), 352-369.

[6] Lin, C.C. AND Y. Y. LAU, On certain eigenvalue problems occuring in physical systems, Adv. in Math., 22 (1976), 120-128.

[7] Lin, C.C. AND Y. Y. LAU, Density wave theory of spiral structure of galaxies, Studies in Appl. Math., 60 (1979), 97-163.

[8] Mark, J.W.K., On density waves in galaxies, I, II, III, IV, V. Astrophys. J., 193 (1974) 539-559, 203 (1976) 81-96, 205 (1976) 363-378, 206 (1976) 418-434, 212 (1977) $645-658$.

[9] Nishimoto, T., Global dispersion relation for density waves in a certain simplified model of a disk shaped galaxy. Studies in Appl. Math., 60 (1979) 11-26.

[10] Pannatoni, R.F. AND Y.Y. LAU, Unstable spiral modes in disk galaxies: A general fluid dynamical theory with some preliminary results. Proc. Natl. Acad. Sci. USA, 76 (1979) 4-7.

[11] Rohlfs, K., Lectures on density wave theory, Lecture Notes in Physics 69 Springer-Verlag (1977).

[12] WAsow, W., Asymptotic expansions for ordinary differential equations. Interscience 1965.

Department of Mathematics

TOKyo Institute of TEChNOLOGy 